\title{
Syntheses of asymmetric 2-benzopyrans. The influence of aromatic halogen substituents on the intramolecular cyclisation of enantiopure tethered phenolic lactaldehydes
}

\author{
Robin G. F. Giles, ${ }^{\mathrm{a},}{ }^{*}$ Ivan R Green, ${ }^{\mathrm{b}}$ Francois J. Oosthuizen, ${ }^{\mathrm{a}}$ and C. Peter Taylor ${ }^{\mathrm{a}}$ \\ ${ }^{a}$ Chemistry Department, Murdoch University, Murdoch, WA 6150, Australia \\ ${ }^{b}$ Chemistry Department, University of the Western Cape, Bellville, 7530, South Africa \\ E-mail: R.Giles@Murdoch.edu.au
}

This paper is dedicated to Professor J. R. Bull on the occasion of his $65^{\text {th }}$ birthday

\begin{abstract}
The syntheses of enantiopure brominated and chlorinated phenolic lactaldehydes are described as well as an investigation into their cyclisation to form the corresponding 2-benzopyran-4,5-diols. It is found that the choice of halogen is important in these processes.
\end{abstract}

Keywords: 2-Benzopyrans, halogenated phenols, lactaldehyde, enantiopure

\section{Introduction}

In model studies ${ }^{1}$ involving the assembly of monochiral 2-benzopyrans as intermediates in natural product synthesis, we established a convenient route from the benzyl alcohol 1 and ethyl (S)-lactate 2 to the enantiopure $a S, 2 S$ phenolic lactaldehyde 3 in a sequence of reactions that maintained the stereochemical integrity at the asymmetric centre. This lactaldehyde was converted into the 2-benzopyran-4,5-diol 4 in high yield in a completely diastereoselective reaction using titanium tetraisopropoxide (Scheme 1). For the assembly of the natural products themselves $^{2}$ the regioselectively halogenated tethered phenolic lactaldehyde $\mathbf{5}$ or $\mathbf{6}$ was required in the enantiomeric $a R, 2 R$ form, derived from the more expensive methyl $(R)$-lactate. The purpose of this halogen was to control the regiochemistry of a subsequent reaction. ${ }^{3}$ We now report the syntheses of each of these aldehydes $\mathbf{5}$ and $\mathbf{6}$ and an investigation into their cyclisations to form the desired 2-benzopyrans $\mathbf{7}$ and $\mathbf{8}$. 
<smiles>COc1ccc(Cc2ccccc2)cc1C(C)O</smiles>

1<smiles>COc1ccc(O)cc1[C@@H](C)O[C@H](C)C=O</smiles>

3<smiles>COc1ccc(O)c2c1[C@H](C)O[C@@H](C)[C@H]2O</smiles>

4

Scheme 1<smiles>[X]c1cc(O)cc([C@@H](C)O[C@@H](C)C=O)c1OC</smiles>

5
$\mathrm{X}=\mathrm{Cl}$
$\mathrm{X}=\mathrm{Br}$<smiles>[X]c1cc(O)c2c(c1OC)[C@H](C)O[C@H](C)[C@H]2O</smiles>

7

\section{Results and Discussion}

\section{Syntheses of the halogenated benzyl alcohols 16 and 17}

The starting material chosen for the assembly of the chlorinated alcohol 16 was commercially available ortho-chlorophenol. This was converted into the known 2-bromo-6-chloro-1,4benzoquinone 9 through dibromination ${ }^{4}$ followed by oxidation. ${ }^{5}$ Reduction with sodium dithionite afforded the corresponding hydroquinone, which was selectively monobenzylated at the less hindered hydroxyl group to yield the 4-benzyloxy derivative 10 as the major product (42\%), together with the dibenzylated product 11 in 20\% yield. Methylation of the remaining phenolic group of the monobenzyl ether 10 afforded the differentially protected hydroquinone dialkyl ether 12 in 85\% yield. Lithium-halogen exchange using butyl lithium followed by reaction with acetaldehyde replaced the bromine atom selectively with the required hydroxyethyl substituent to provide the target chlorinated benzyl alcohol 16 in a yield of $63 \%$, together with the debrominated starting material 5-benzyloxy-2-methoxychlorobenzene (34\%). 
<smiles>O=C1C=C(Cl)C(=O)C(Br)=C1</smiles>

9<smiles>[R]Oc1cc(Cl)c(O[Ga])c(Br)c1</smiles>

$10 \mathrm{R}^{1}=\mathrm{H}, \mathrm{R}^{2}=\mathrm{Bn}$

$11 \mathrm{R}^{1}=\mathrm{R}^{2}=\mathrm{Bn}$

$12 \mathrm{R}^{1}=\mathrm{Me}, \mathrm{R}^{2}=\mathrm{Bn}$<smiles>[R]Oc1c([X])cc(Cc2ccccc2)cc1C(C)=O</smiles>

$13 \mathrm{X}=\mathrm{R}=\mathrm{H}$

$14 \mathrm{X}=\mathrm{Br}, \mathrm{R}=\mathrm{H}$

$15 \mathrm{X}=\mathrm{Br}, \mathrm{R}=\mathrm{Me}$

An alternative strategy was employed to assemble the corresponding brominated alcohol 17. 5-Benzyloxy-2-hydroxyacetophenone $\mathbf{1 3}^{1}$ was regioselectively brominated to afford the 3-bromo derivative 14 in 78\% yield. The phenolic hydroxyl group was methylated to afford the differentially protected hydroquinone dialkyl ether 15 in $94 \%$ yield, and this was reduced with sodium borohydride to give the required brominated benzyl alcohol $\mathbf{1 7}$ in $96 \%$ yield. In this last reaction, on the other hand, an excess of lithium aluminium hydride also removed the aromatic bromine atom.

\section{Syntheses of the brominated lactaldehydes 27 and 30 and the chlorinated lactaldehydes 35}

The brominated benzyl alcohol $\mathbf{1 7}$ was converted into the corresponding benzyl bromide $\mathbf{1 8}$ in $78 \%$ using phosphorus tribromide. The use of silver trifluoroacetate and ethyl (S)-lactate to convert this product into $O$-benzyl-protected lactates ${ }^{1}$ gave only moderate yields of the desired products. A considerable improvement was achieved by conversion of the brominated benzyl alcohol 17 into the corresponding trichloroacetimidate 19 in 77\% yield. Reaction of this with methyl $(R)$-lactate in the presence of catalytic quantities of boron trifluoride diethyl ether gave the inseparable mixture of benzyl-protected methyl lactates 21 in 64\% yield, while with the alternative ester isobutyl $(R)$-lactate the corresponding inseparable mixture of esters 22 was obtained in $79 \%$ yield.
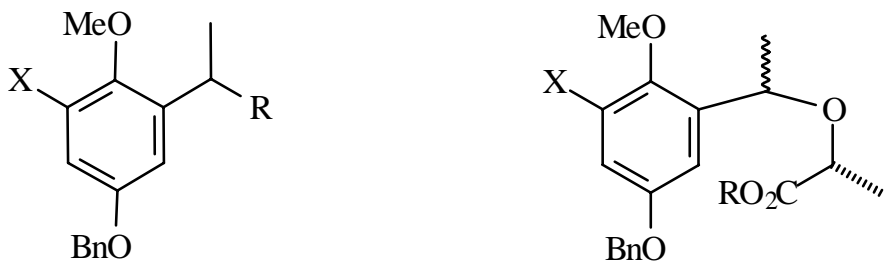

$$
\begin{aligned}
& 16 X=\mathrm{Cl}, \mathrm{R}=\mathrm{OH} \\
& 17 \mathrm{X}=\mathrm{Br}, \mathrm{R}=\mathrm{OH} \\
& \mathbf{1 8} \mathrm{X}=\mathrm{R}=\mathrm{Br} \\
& 19 \mathrm{X}=\mathrm{Br}, \mathrm{R}=\mathrm{OC}(=\mathrm{NH}) \mathrm{CCl}_{3} \\
& \mathbf{2 0} \mathrm{X}=\mathrm{Cl}, \mathrm{R}=\mathrm{OC}(=\mathrm{NH}) \mathrm{CCl}_{3}
\end{aligned}
$$

$21 \mathrm{X}=\mathrm{Br}, \mathrm{R}=\mathrm{Me}$

$22 \mathrm{X}=\mathrm{Br}, \mathrm{R}=\mathrm{CH}_{2} \mathrm{CHMe}$

$23 \mathrm{X}=\mathrm{Cl}, \mathrm{R}=\mathrm{CH}_{2} \mathrm{CHMe}_{2}$

The synthesis of these benzyl-epimeric pairs of esters 21 and 22, as well as the pair from the model study, ${ }^{1}$ gave, in each case, the two diastereoisomers in approximately equal proportions, 
whereas only the $\alpha R$ configuration was required. It was anticipated that the nucleophilic substitution process involving the replacement of the activating trichloroacetimidate group by the nucleophilic lactate alcohol oxygen would be more $S_{N} 1$ - than $S_{N} 2$-like in character, particularly since the benzylic carbocation produced from the trichloroacetimidate would enjoy further stabilization from the ortho-methoxy substituent. The ratio of the diastereoisomeric benzylprotected lactates would therefore not be expected to alter if the starting imidate were monochiral if the reaction proceeded via an $\mathrm{S}_{\mathrm{N}} 1$ mechanism, but would if an $\mathrm{S}_{\mathrm{N}} 2$ mechanism were involved, in which the nucleophilic alcohol displaced the departing imidate. Any improvement in the proportion of the $\alpha R$ stereoisomer would nevertheless be an advantage and the trichloroacetimidate was therefore prepared as almost exclusively a single enantiomer. It was recognized that, for an $S_{N} 2$ process, the $(\alpha S)$ enantiomer of the alcohol $\mathbf{1 7}$ would be required to yield the $\alpha R, 2 R$ lactate through inversion, but only the less expensive (S)-enantiomer of the required reducing agent was immediately available in our laboratory and this would produce the $\alpha R$ enantiomer, so this was used as a model. The acetophenone $\mathbf{1 5}$ was therefore subjected to enantioselective reduction using freshly prepared (S)-oxazaborolidine (CBS-catalyst) and borane-dimethyl sulphide complex, ${ }^{6}$ to give the $(\alpha R)$ enantiomer 24 of the benzyl alcohol 17. ${ }^{7} \mathrm{~A}$ $98 \%$ yield was obtained when the reaction was undertaken at $-23{ }^{\circ} \mathrm{C}$, and the enantiomeric excess was determined as $94 \%$. A lower enantiomeric excess, $82 \%$, was obtained when the reaction was performed at the higher temperature of $0{ }^{\circ} \mathrm{C}$. This was then converted as before into the enantiopure $(\alpha R)$ trichloroacetimidate 25 . When this was reacted with isobutyl $(R)$-lactate an approximately 1:1 mixture of the benzyl-protected lactates 22 was obtained (Scheme 2). This supported an $\mathrm{S}_{\mathrm{N}} 1$-like carbocation for this process.

15

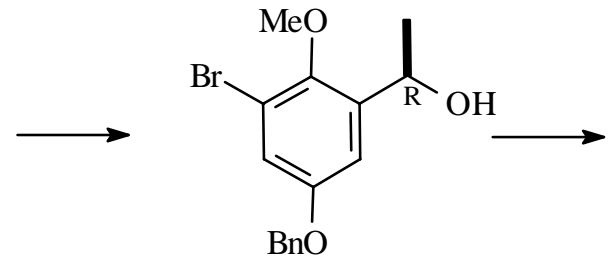

24

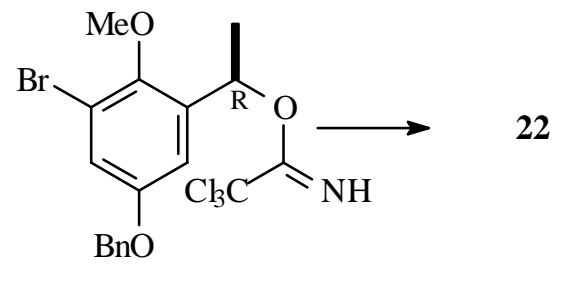

25

\section{Scheme 2}

The individual lactaldehydes $\mathbf{2 7}$ and $\mathbf{3 0}$ were the next targets, and these were best obtained directly as a mixture (66\% yield) through reduction of the mixture of esters 22 with diisobutyl aluminium hydride, together with the individual alcohols 26 (9\%) and 29 (10\%). The mixture of aldehydes was, however, inseparable, and so the ester mixture 22 was reduced with lithium borohydride to afford the mixture of alcohols, which was separated chromatographically to give the individual diastereoisomers 26 (36\%) and 29 (33\%). Lithium aluminium hydride was not used in this reaction in view of its removal of the aromatic bromine atom in the reduction of the ketone $\mathbf{1 5}$ as described above. Each of these alcohols $\mathbf{2 6}$ and $\mathbf{2 9}$ was oxidized separately to the 
corresponding aldehyde using Swern's method, ${ }^{8}$ the aldehyde 27 being obtained in a yield of $72 \%$ and the diastereoisomer 30 in $86 \%$.

For the corresponding chloro compounds, the same sequence of reactions was followed as used above for their brominated analogues. Thus the alcohol $\mathbf{1 6}$ was converted into its trichloroacetimidate 20, and thence into the inseparable mixture of benzyl-protected lactate esters 23. Reduction of this mixture with lithium aluminium hydride afforded the mixture of alcohols 34. Unlike the corresponding brominated alcohols $\mathbf{2 6}$ and $\mathbf{2 9}$ above and those used in the model study, ${ }^{1}$ the individual chlorinated alcohols of the diastereoisomeric mixture $\mathbf{3 4}$ could not be separated chromatographically. They were therefore oxidized as a mixture, using the Swern method, to the related mixture of aldehydes 35 .

\section{Generation of the phenolic lactaldehydes and an investigation into their cyclisations}

The mixture of chlorinated phenolic lactaldehydes 36 was generated through benzylic hydrogenolysis of the corresponding benzyl ethers 35 . These phenolic materials were unstable on standing and so were subjected immediately as a mixture of diastereoisomers to treatment with titanium tetraisopropoxide under ultrasonic radiation, conditions which had led in the model studies $^{1}$ to high yields of 2-benzopyran-4,5-diols, for each of the starting materials $\mathbf{3}$ and its benzyl epimer. In the present study, however, where the sole difference in the substrates (other than that the model compounds were the enantiomers of the aldehydes 36) was their regioselective chlorination, no cyclisation product 7 was observed under identical reaction conditions, only starting materials being recovered. 
21 or 22

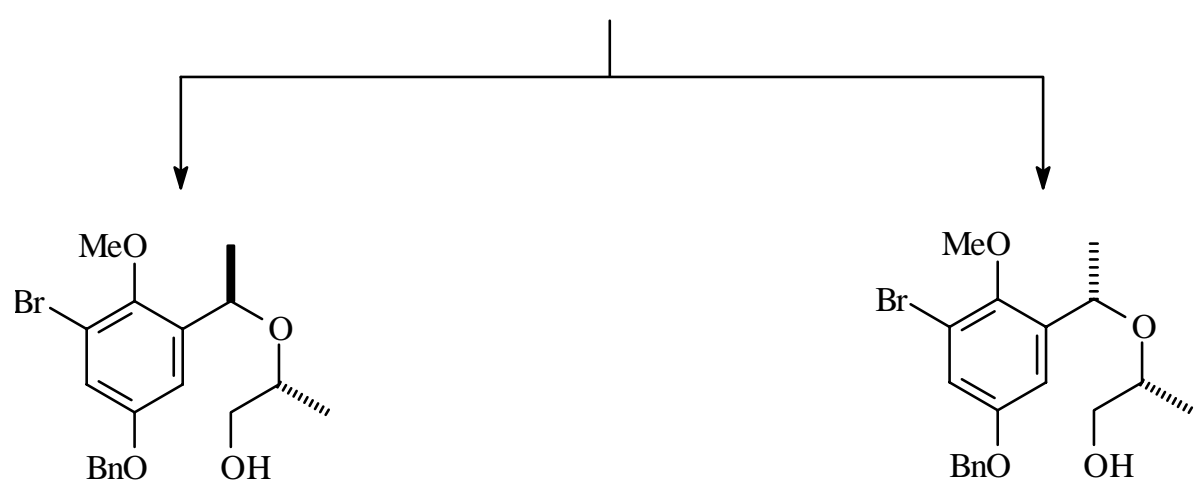<smiles>COc1c(Br)cc(OCc2ccccc2)cc1[C@H](C)O[C@@H](C)C=O</smiles><smiles>[R]Oc1cc(Br)c(OC)c([C@@H](C)O[C@H](C)C=O)c1</smiles>

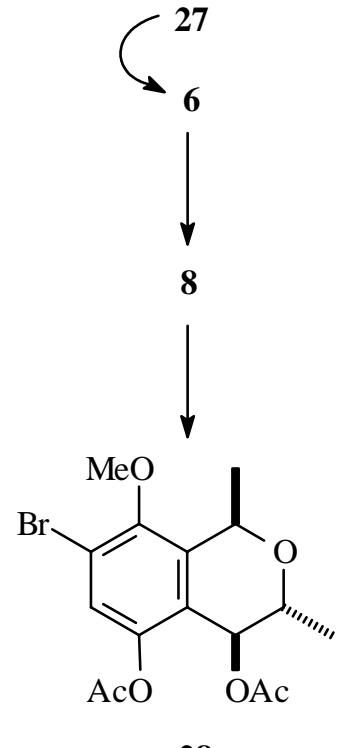<smiles>[R]CC[C@H]([R])C</smiles>

Hydrogenolysis of the brominated benzyl ether 27 afforded the unstable brominated phenolic lactaldehyde $\mathbf{6}$, which was immediately cyclised with titanium tetraisopropoxide under ultrasonic radiation, whereupon the product 7-bromo-2-benzopyran-4,5-diol 8 was immediately converted into the diacetate 28 in an overall yield for the two steps of 74\%, thus averaging $86 \%$ for each step. The cyclisation step was completely diastereoselective and the stereochemistry of the 2benzopyran-4,5-diol $\mathbf{8}$ and its diacetate $\mathbf{2 8}$ were readily identified from their ${ }^{1} \mathrm{H}$ NMR spectra; in particular, for the diacetate from the coupling constant between $3-\mathrm{H}$ and $4-\mathrm{H}$, which was found 
to be $5.0 \mathrm{~Hz}$, and the chemical shift of $3-\mathrm{H}$ was $\delta 4.10$. These values agree closely with those (4.8 Hz and $\delta 4.11$ ) found for the model diacetate of the diol $4,{ }^{1}$ which is the debrominated enantiomer of the diacetate $\mathbf{2 8}$.<smiles>COc1c(Cl)cc(Cc2ccccc2)cc1[C@@H](C)O[C@H](C)CO</smiles>

34<smiles>[R9]c1cc(Cl)c(OC)c([C@@H](C)O[C@H](C)C=O)c1</smiles>

$35 \mathrm{R}=\mathrm{Bn}$

$36 \mathrm{R}=\mathrm{H}$

Hydrogenolysis of the benzyl epimeric lactaldehyde $\mathbf{3 0}$ gave the phenol 31, which was immediately cyclised to the mixture of C-4 epimeric 4,5-diols ${ }^{1} 32$ and 33 in a ratio of 1:1.

\section{Conclusions}

The successful diastereoselective cyclisation of tethered phenolic lactaldehydes to form enantiopure 2-benzopyran-4,5-diols using titanium tetraisopropoxide under ultrasonic radiation is influenced by electron availability on the aromatic ring. Chlorine substitution of this ring para to the desired reaction site prevents cyclisation, whereas the less electronegative bromine facilitates ready ring-closure. Bromine is therefore the halogen of choice in completing the syntheses of the desired natural products.

\section{Experimental Section}

General Procedures. Nuclear magnetic resonance spectra were recorded using either a Hitachi R24B spectrometer $\left({ }^{1} \mathrm{H} 60 \mathrm{MHz}\right)$, a Bruker AM-300 spectrometer $\left({ }^{1} \mathrm{H} 300 \mathrm{MHz},{ }^{13} \mathrm{C} 75.5 \mathrm{MHz}\right)$ or a Bruker Avance DPX-300 spectrometer $\left({ }^{1} \mathrm{H} 300 \mathrm{MHz},{ }^{13} \mathrm{C} 75.5 \mathrm{MHz}\right)$. All spectra were run on the Bruker AM-300 spectrometer or the Bruker Avance DPX-300 spectrometer unless otherwise stated. All ${ }^{1} \mathrm{H}$ spectra were recorded at ambient temperature in deuterochloroform $\left(\mathrm{CDCl}_{3}\right)$ using tetramethylsilane (TMS) as an internal standard. In the ${ }^{13} \mathrm{C}$ NMR spectra, assignments of signals with the same superscript are interchangeable. Mass spectra were recorded on either a Hewlett Packard 5986 spectrometer at $35 \mathrm{eV}$, or on a Perkin Elmer ITD Ion Trap Detector spectrometer at $55 \mu \mathrm{A}$ with automatic gain control. High resolution mass spectra were recorded on a VG_Autospec High Resolution Mass Spectrometer at the University of Western Australia. Infrared spectra were recorded as thin films between $\mathrm{KBr}$ plates for oils and as $\mathrm{KBr}$ discs for solids using a Perkin Elmer 1720-X Fourier Transform Spectrometer. Melting 
points are uncorrected and were recorded on a Reichert hot stage apparatus. Optical rotations were recorded for chloroform solutions of $c 1.0$ at $20^{\circ} \mathrm{C}$ using an Optical Activity PolAAr 2001 polarimeter. The sonication bath used was a Branson B3200-E4 operating at a frequency of 44$50 \mathrm{kHz}$. Kugelrohr refers to a Kugelrohr distillation apparatus. Elemental analysis were carried out by either the Australian National University Analytical Service Unit or by the Canadian Microanalytical Service Ltd. Column chromatography was performed on columns prepared as slurries of Merck silica gel 60 (70-230 mesh) in the eluent. Preadsorption was carried out on Merck silica gel 60 (35-70 mesh). Radial chromatography was performed using Merck silica gel $60 \mathrm{PF}_{254}$. Preparative layer chromatography was performed on glass plates coated with Carmag silica gel as a $0.3 \mathrm{~mm}$ thick layer, while thin layer chromatography was carried out on aluminium plates coated with Merck Kieselgel $60 \mathrm{~F}_{254}$. Petroleum ether refers to the fraction of boiling point $65{ }^{\circ} \mathrm{C}$ to $70{ }^{\circ} \mathrm{C}$. All solvents were purified by distillation and, if necessary, were dried according to standard methods. The amount of residual water present in solvents was determined using a Metrohm Karl Fischer Coulometer 684.

4-Benzyloxy-2-bromo-6-chlorophenol (10). A solution of quinone 9 (0.70 g, $3.16 \mathrm{mmol})$ in diethyl ether $(100 \mathrm{~mL})$ and dichloromethane $(50 \mathrm{~mL})$ was shaken with a solution of sodium dithionite (3.64 g, $18.94 \mathrm{mmol})$ in water $(40 \mathrm{~mL})$ for $2 \mathrm{~min}$. The organic layer was washed with water, dried (magnesium sulfate) and concentrated to give crude hydroquinone ( $0.65 \mathrm{~g}, 2.91$ mmol crude). A solution of potassium carbonate $(0.40 \mathrm{~g}, 2.89 \mathrm{mmol})$ in dry acetone $(10 \mathrm{~mL})$ was heated under reflux for $20 \mathrm{~min}$. Benzyl bromide $(0.40 \mathrm{~g}, 2.34 \mathrm{mmol})$ was added and the reaction heated under reflux for a further $5 \mathrm{~min}$. A solution of the crude hydroquinone $(0.65 \mathrm{~g}, 2.91 \mathrm{mmol}$ crude) in dry acetone (10 mL) was added and the mixture heated under reflux for a further $2 \mathrm{~h}$. The mixture was cooled, filtered and concentrated to give a crude mixture that was chromatographed (radial, 5\% ethyl acetate-petroleum ether) to give two products. The higher $\mathrm{R}_{\mathrm{f}}$ product was identified as 2-bromo-6-chloro-1,4-dibenzyloxybenzene 11 (0.26 g, 20\%) (Found: $\mathrm{M}^{+}$, 401.9928. $\mathrm{C}_{20} \mathrm{H}_{16}{ }^{79} \mathrm{Br}^{35} \mathrm{ClO}_{2}$ requires $\mathrm{M}$, 402.0022); $v_{\max } / \mathrm{cm}^{-1} 2876(\mathrm{C}-\mathrm{H}), 1594(\mathrm{C}=\mathrm{C})$, $1449(\mathrm{C}=\mathrm{C}) ; \delta_{\mathrm{H}} 4.91$ and 4.94 (each $2 \mathrm{H}, \mathrm{s}, 1-\mathrm{OCH}_{2}$ and 4- $\mathrm{OCH}_{2}$ ), 6.96 and 7.08(each $1 \mathrm{H}, \mathrm{d}, J$ 2.9, 3-H and 5-H), 7.25-7.39 (8H, m, $\mathrm{Ar}^{\prime}-\mathrm{H}$ and $\left.\mathrm{Ar}^{\prime \prime}-\mathrm{H}\right), 7.52-7.56\left(2 \mathrm{H}, \mathrm{m}, 2^{\prime}-\mathrm{H}\right.$ and 6'-H on 4$\mathrm{OCH}_{2} \mathrm{Ph}$ substituent); $\delta_{\mathrm{C}} 70.6\left(1-\mathrm{OCH}_{2}\right),{ }^{\mathrm{a}} 74.8\left(4-\mathrm{OCH}_{2}\right),{ }^{\mathrm{a}} 116.1(\mathrm{C}-3),{ }^{\mathrm{b}} 118.2(\mathrm{C}-5),{ }^{\mathrm{b}} 118.7$ (C6), 127.4, 128.21, 128.22, 128.3 and 128.6 (C-2' and C-6', C-3' and C-5', C-4', C-2"' and C-6"', C-4", C-3" and C-5"), 129.4 (C-2), 135.8 (C-1'), ${ }^{\mathrm{d}} 136.4$ (C-1"), ${ }^{\mathrm{d}} 146.0$ (C-1), ${ }^{\mathrm{e}} 155.2$ (C-4), ${ }^{\mathrm{e}} \mathrm{m} / \mathrm{z}$ 404* [ $\mathrm{M}^{+}\left(\mathrm{C}_{20} \mathrm{H}_{16}{ }^{79} \mathrm{Br}^{37} \mathrm{ClO}_{2}\right.$ or $\left.\left.\mathrm{C}_{20} \mathrm{H}_{16}{ }^{81} \mathrm{Br}^{35} \mathrm{ClO}_{2}\right), 0.4 \%\right], 402$ [M $\left.\mathrm{M}^{+}\left(\mathrm{C}_{20} \mathrm{H}_{16}{ }^{79} \mathrm{Br}^{35} \mathrm{ClO}_{2}\right), 0.5\right], 92$ (14), 91 (100), 65 (20). (* Peak 406 corresponding to $\mathrm{C}_{20} \mathrm{H}_{16}{ }^{81} \mathrm{Br}^{37} \mathrm{ClO}_{2}$ was not observed due to the intensity being too low, corresponding as it would to about $0.1 \%$ ). The lower $\mathrm{R}_{\mathrm{f}}$ product was identified as the title compound 10 (0.42 g, 42\%) (Found: $\mathrm{M}^{+}-1$, 310.9487. $\mathrm{C}_{13} \mathrm{H}_{10}{ }^{79} \mathrm{Br}^{35} \mathrm{ClO}_{2}$ requires M-1, 310.9474); $v_{\max } / \mathrm{cm}^{-1} 3422(\mathrm{O}-\mathrm{H}), 2920(\mathrm{C}-\mathrm{H}), 1594(\mathrm{C}=\mathrm{C}) ; \delta_{\mathrm{H}} 4.96\left(2 \mathrm{H}, \mathrm{s}, \mathrm{CH}_{2}\right)$, 5.90 (1H, br. s, OH), 6.79 and 6.90 (each 1H, d, $J$ 2.9, 3-H and 5-H), 7.34-7.42 (3H, m, 3'-H, 4'$\mathrm{H}$ and 5'-H), 7.56-7.58 (2H, 2'-H and 6'-H); $\delta_{\mathrm{c}} 75.2\left(\mathrm{CH}_{2}\right), 116.8$ (C-3), ${ }^{\mathrm{a}} 118.6$ (C-6), 118.9 (C- 
5), ${ }^{\mathrm{a}} 128.5$ (C-2', C-4' and C-6'), ${ }^{\mathrm{b}} 128.7$ (C-3' and C-5'), 129.4 (C-2), 136.0 (C-1'), 145.5 (C-1), ${ }^{\mathrm{c}}$ 152.6 (C-4); ${ }^{\mathrm{C}} \mathrm{m} / \mathrm{z} 316\left[\mathrm{M}^{+}\left(\mathrm{C}_{13} \mathrm{H}_{10}{ }^{81} \mathrm{Br}^{37} \mathrm{ClO}_{2}\right), 0.6 \%\right], 314\left[\mathrm{M}^{+}\left(\mathrm{C}_{13} \mathrm{H}_{10}{ }^{79} \mathrm{Br}^{37} \mathrm{ClO}_{2}\right.\right.$ or $\mathrm{C}_{13} \mathrm{H}_{10}{ }^{81} \mathrm{Br}^{35} \mathrm{ClO}_{2}$ ), 2.5], 312 [ $\left.\mathrm{M}^{+}\left(\mathrm{C}_{13} \mathrm{H}_{10}{ }^{79} \mathrm{Br}^{35} \mathrm{ClO}_{2}\right), 1.9\right], 91$ (100), 65 (11).

4-Benzyloxy-2-bromo-6-chloro-1-methoxybenzene (12). Potassium carbonate (6.98 g, 50.5 mmol) was added to a solution of phenol 10 (3.96 g, $12.6 \mathrm{mmol}$ ) and dimethyl sulfate (3.98 g, $31.6 \mathrm{mmol})$ in dry acetone $(120 \mathrm{~mL})$ and the mixture heated under reflux for $2 \mathrm{~h}$. It was then cooled, filtered and concentrated. The residue was dissolved in diethyl ether and stirred with aqueous sodium hydroxide (10\%) for $2 \mathrm{~h}$. The organic layer was washed with water, dried (magnesium sulfate), concentrated and the residue recrystallised to give product $\mathbf{1 2}$ as white crystals (3.51 g, 85\% yield) mp 73.5-76.5 ${ }^{\circ} \mathrm{C}$ (from hexane) (Found: C, 51.2; H, 3.65. $\mathrm{C}_{14} \mathrm{H}_{12} \mathrm{BrClO}_{2}$ requires C, 51.35; $\left.\mathrm{H}, 3.7 \%\right) ; v_{\max } / \mathrm{cm}^{-1} 2959(\mathrm{C}-\mathrm{H}), 1596(\mathrm{C}=\mathrm{C}), 1556(\mathrm{C}=\mathrm{C})$, $1474(\mathrm{C}=\mathrm{C}), 1463(\mathrm{C}=\mathrm{C}) ; \delta_{\mathrm{H}} 3.76\left(3 \mathrm{H}, \mathrm{s}, \mathrm{OCH}_{3}\right), 4.97\left(2 \mathrm{H}, \mathrm{s}, \mathrm{CH}_{2}\right), 6.92$ and 7.03 (each $1 \mathrm{H}, \mathrm{d}, J$ 3.0, 3-H and 5-H), 7.32-7.43 (3H, m, 3'-H, 4'-H and 5'-H), 7.55-7.60 (2H, m, $2^{\prime}-\mathrm{H}$ and $\left.6^{\prime}-\mathrm{H}\right) ; \delta_{\mathrm{C}}$ $56.8\left(\mathrm{CH}_{3}\right), 75.8\left(\mathrm{CH}_{2}\right), 116.2(\mathrm{C}-3),{ }^{\mathrm{a}} 118.3(\mathrm{C}-5),{ }^{\mathrm{a}} 119.7$ (C-6), $129.2\left(\mathrm{C}-4^{\prime}\right), 129.3\left(\mathrm{C}-2^{\prime}\right.$ and C6'), 129.4 (C-3' and C-5'), ${ }^{\mathrm{b}} 130.4$ (C-2), 137.4 (C-1'), 146.7 (C-1), ${ }^{\mathrm{c}} 157.1$ (C-4); ${ }^{\mathrm{c}} \mathrm{m} / \mathrm{z} 329$ [M $\mathrm{M}^{+}-1$ $\left.\left(\mathrm{C}_{14} \mathrm{H}_{11}{ }^{81} \mathrm{Br}^{37} \mathrm{ClO}_{2}\right), 1 \%\right], 327\left[\mathrm{M}^{+}-1\left(\mathrm{C}_{14} \mathrm{H}_{11}{ }^{79} \mathrm{Br}^{37} \mathrm{ClO}_{2}\right.\right.$ or $\left.\left.\mathrm{C}_{14} \mathrm{H}_{11}{ }^{81} \mathrm{Br}^{35} \mathrm{ClO}_{2}\right), 2.1\right], 326\left[\mathrm{M}^{+}-1\right.$ $\left(\mathrm{C}_{14} \mathrm{H}_{11}{ }^{79} \mathrm{Br}^{35} \mathrm{ClO}_{2}\right.$ ), 2], 247 (18), 93 (11), 92 (51), 91 (100), 65 (16).

5'-Benzyloxy-3'-bromo-2'-hydroxyacetophenone (14). Bromine (0.198 g, $1.24 \mathrm{mmol})$ was added to a solution of $5^{\prime}$-benzyloxy-2'-hydroxyacetophenone $13^{1,9}(0.300 \mathrm{~g}, 1.24 \mathrm{mmol})$ and pyridine $(0.249 \mathrm{~g}, 3.15 \mathrm{mmol})$ in dry dichloromethane $(7 \mathrm{~mL})$ at $0{ }^{\circ} \mathrm{C}$. The solution was stirred at $0{ }^{\circ} \mathrm{C}$ for $5 \mathrm{~min}$ and then at room temperature for $3 \mathrm{~h}$. The reaction was quenched with hydrochloric acid $(1 \mathrm{M})$ and the mixture was extracted with dichloromethane. The organic extracts were washed with hydrochloric acid $(1 \mathrm{M})$ and saturated sodium chloride. The residue upon workup was chromatographed (50\% ethyl acetate-petroleum ether) to give the product $\mathbf{1 4}$ (0.312 g, 78\%) as pale yellow needles mp 87-88 ${ }^{\circ} \mathrm{C}$ (from hexane) (Found: $\mathrm{C}, 56.1 ; \mathrm{H}, 4.0$. $\mathrm{C}_{15} \mathrm{H}_{13} \mathrm{BrO}_{3}$ requires C, 56.1; $\left.\mathrm{H}, 4.1 \%\right) ; v_{\max } / \mathrm{cm}^{-1} 3583(\mathrm{O}-\mathrm{H}), 1647(\mathrm{C}=\mathrm{O}), 1614(\mathrm{C}=\mathrm{C}), 1446$ $(\mathrm{C}=\mathrm{C}) ; \delta_{\mathrm{H}} 2.56\left(3 \mathrm{H}, \mathrm{s}, \mathrm{CH}_{3}\right), 5.00\left(2 \mathrm{H}, \mathrm{s}, \mathrm{CH}_{2}\right), 7.22(1 \mathrm{H}, \mathrm{d}, J$ J 2.9, 4'-H), 7.33-7.40 (5H, m, PhH), $7.43\left(1 \mathrm{H}, \mathrm{d}, J\right.$ 2.9, 6'-H), $12.44(1 \mathrm{H}, \mathrm{s}, \mathrm{OH}) ; \delta_{\mathrm{C}} 26.7\left(\mathrm{CH}_{3}\right), 71.3\left(\mathrm{CH}_{2}\right), 112.0\left(\mathrm{C}-3^{\prime}\right), 115.4$ (C-6'), 119.6 (C-1'), 125.9 (C-4'), 127.6 (C-2" and C-6"), 128.3 (C-4" ), 128.7 (C-3" and C-

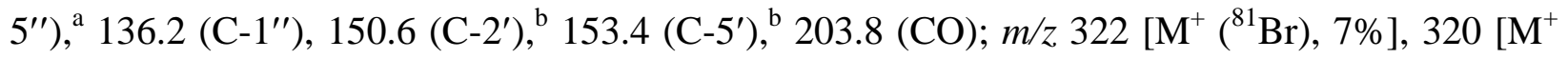
$\left.\left({ }^{79} \mathrm{Br}\right), 7\right], 91$ (100), 65 (14).

5'-benzyloxy-3'-bromo-2'-methoxyacetophenone (15). Potassium carbonate (3.03 g, 21.9 mmol) was added to a stirred solution of dimethyl sulfate ( $2.76 \mathrm{~g}, 21.9 \mathrm{mmol}$ ) and compound 14 $(2.81 \mathrm{~g}, 8.8 \mathrm{mmol})$ in dry acetone $(60 \mathrm{~mL})$. The mixture was heated under reflux for $17 \mathrm{~h}$, then cooled, filtered and the filtrate concentrated. The residue was dissolved in dichloromethane and washed with water and saturated aqueous sodium chloride. After workup, the crude compound was chromatographed (10-20\% ethyl acetate-petroleum ether) to give the title compound $\mathbf{1 5}$ (2.76 g, 94\%) as an oil (Found: C, 57.85; $\mathrm{H}, 4.85 . \mathrm{C}_{16} \mathrm{H}_{15} \mathrm{BrO}_{3}$ requires $\mathrm{C}, 57.5 ; \mathrm{H}, 4.55 \%$ ); $v_{\max } / \mathrm{cm}^{-1} 2939(\mathrm{C}-\mathrm{H}), 1685(\mathrm{C}=\mathrm{O}), 1596(\mathrm{C}=\mathrm{C}), 1558(\mathrm{C}=\mathrm{C}), 1467(\mathrm{C}=\mathrm{C}) ; \delta_{\mathrm{H}} 2.62(3 \mathrm{H}, \mathrm{s}$, $\left.\mathrm{COCH}_{3}\right), 3.81\left(3 \mathrm{H}, \mathrm{s}, \mathrm{OCH}_{3}\right), 5.02\left(2 \mathrm{H}, \mathrm{s}, \mathrm{CH}_{2}\right), 7.17\left(1 \mathrm{H}, \mathrm{d}, J 3.1,4^{\prime}-\mathrm{H}\right), 7.33\left(1 \mathrm{H}, \mathrm{d}, J 3.1,6^{\prime}-\right.$ 
H), 7.33-7.39 (5H, m, Ph-H); $\delta_{\mathrm{c}} 30.4\left(\mathrm{COCH}_{3}\right), 62.5\left(\mathrm{OCH}_{3}\right), 70.7\left(\mathrm{CH}_{2}\right), 114.3\left(\mathrm{C}-6^{\prime}\right), 118.7$ (C-3'), 123.9 (C-4'), 127.5 (C-2" and C-6"'), ${ }^{a} 128.3$ (C-4"), 128.7 (C-3" and C-5"), ${ }^{a} 134.8$ (C-1'),

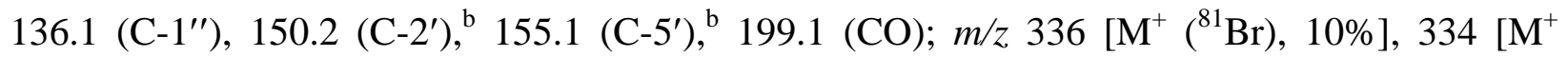
$\left.\left({ }^{79} \mathrm{Br}\right), 11\right], 91$ (100), 65 (10).

1-(5'-Benzyloxy-3'-chloro-2'-methoxyphenyl)ethanol (16). Butyl lithium (0.161 mL, 1.90M in cyclohexane) was added to a solution of bromobenzene $12(0.100 \mathrm{~g}, 0.30 \mathrm{mmol})$ in tetrahydrofuran $(10 \mathrm{~mL})$ at $-78{ }^{\circ} \mathrm{C}$. The mixture was stirred at $-78{ }^{\circ} \mathrm{C}$ for $15 \mathrm{~min}$, then acetaldehyde $(1 \mathrm{~mL})$ was added and the reaction mixture stirred for a further $15 \mathrm{~min}$ at $-78{ }^{\circ} \mathrm{C}$. The reaction was quenched with water, warmed to room temperature and extracted with diethyl ether. The extracts were dried (magnesium sulfate), concentrated and chromatographed (radial, $10-50 \%$ ethyl acetate-petroleum ether) to give two products. The higher $\mathrm{R}_{\mathrm{f}}$ product was identified as 4-benzyloxy-2-chloro-1-methoxybenzene (26 mg, 34\%) (Found: C, 67.15; H, 5.35. $\mathrm{C}_{14} \mathrm{H}_{13} \mathrm{ClO}_{2}$ requires $\left.\mathrm{C}, 67.6 ; \mathrm{H}, 5.25 \%\right) ; v_{\max } / \mathrm{cm}^{-1} 2932(\mathrm{C}-\mathrm{H}), 1497(\mathrm{C}=\mathrm{C}), 1457(\mathrm{C}=\mathrm{C}) ; \delta_{\mathrm{H}}$ 3.75 (3H, s, $\left.\mathrm{CH}_{3}\right), 5.08\left(2 \mathrm{H}, \mathrm{s}, \mathrm{CH}_{2}\right), 6.71(1 \mathrm{H}, \mathrm{dd}, J 3.0$ and 9.0, 5-H), $6.89(1 \mathrm{H}, \mathrm{d}, J$ 9.0, 6-H), 6.96 (1H, d, J 3.0, 3-H), 7.31-7.46 (5H, m, Ph-H); $\delta_{\mathrm{C}} 55.8\left(\mathrm{CH}_{3}\right), 72.0\left(\mathrm{CH}_{2}\right), 112.9(\mathrm{C}-3),{ }^{a} 116.0$ (C-5), ${ }^{a} 116.1$ (C-6), ${ }^{a} 124.2$ (C-2), 127.3 (C-2' and C-6'), ${ }^{\mathrm{b}} 127.9$ (C-4'), 128.5 (C-3' and C-5'), 136.8 (C-1'), 148.4 (C-1), ${ }^{\mathrm{c}} 154.2$ (C-4); ${ }^{\mathrm{C}} \mathrm{m} / \mathrm{z} 250$ [M $\left.\left.{ }^{+}{ }^{37} \mathrm{Cl}\right), 2 \%\right], 248$ [ $\left.\mathrm{M}^{+}\left({ }^{35} \mathrm{Cl}\right), 7\right], 157$ (13), 91 (100), 65 (36), 63 (13). The lower $\mathrm{R}_{\mathrm{f}}$ product was identified as product 16 (56 mg, 63\%) (Found: C, 65.45; H, 5.9. $\mathrm{C}_{16} \mathrm{H}_{17} \mathrm{ClO}_{3}$ requires $\mathrm{C}$, 65.65; H, 5.85\%); $v_{\max } / \mathrm{cm}^{-1} 3434(\mathrm{O}-\mathrm{H}), 2933$ $(\mathrm{C}-\mathrm{H}), 1603(\mathrm{C}=\mathrm{C}), 1571(\mathrm{C}=\mathrm{C}), 1473(\mathrm{C}=\mathrm{C}) ; \delta_{\mathrm{H}} 1.40\left(3 \mathrm{H}, \mathrm{d}, J\right.$ 6.4, 1-CH $\mathrm{CH}_{3}, 3.78\left(3 \mathrm{H}, \mathrm{s}, \mathrm{OCH}_{3}\right)$, $4.99\left(2 \mathrm{H}, \mathrm{s}, \mathrm{CH}_{2}\right), 5.01-5.08(1 \mathrm{H}, \mathrm{m}, 1-\mathrm{H}), 6.88$ and 6.91 (each $1 \mathrm{H}, \mathrm{d}, J 3.0,4^{\prime}-\mathrm{H}$ and $6^{\prime}-\mathrm{H}$ ), 7.31-7.47 (5H, m, Ph-H); $\delta_{\mathrm{C}} 23.8(\mathrm{C}-2), 55.7\left(\mathrm{OCH}_{3}\right), 65.1(\mathrm{C}-1), 75.6\left(\mathrm{CH}_{2}\right), 110.4\left(\mathrm{C}-4^{\prime}\right)$, ${ }^{a}$ 114.6 (C-6'), ${ }^{\mathrm{a}} 128.1$ (C-3'), 128.4 (C-2", C-4"' and C-6"), 128.6 (C-3" and C-5"'), 136.7 (C-1"), $141.5\left(\mathrm{C}-1^{\prime}\right), 145.1\left(\mathrm{C}-2^{\prime}\right),{ }^{\mathrm{C}} 156.3\left(\mathrm{C}-5^{\prime}\right) ;{ }^{\mathrm{C}} \mathrm{m} / \mathrm{z} 294$ [M $\left.{ }^{+}\left({ }^{37} \mathrm{Cl}\right), 3 \%\right], 292\left[\mathrm{M}^{+}\left({ }^{35} \mathrm{Cl}\right), 9\right], 186$ [( $\left.\left.{ }^{37} \mathrm{Cl}\right) 10\right], 184$ [( $\left.\left.{ }^{35} \mathrm{Cl}\right) 31\right], 91$ (100), 90 (11), 77(14), 65 (29).

1-(5'-Benzyloxy-3'-bromo-2'-methoxyphenyl)ethanol (17). Sodium borohydride (1.62 g, $42.8 \mathrm{mmol}$ ) was added to a stirred solution of compound 15 (11.95 g, $35.6 \mathrm{mmol})$ in dry ethanol $(240 \mathrm{~mL})$. The reaction mixture was stirred for $40 \mathrm{~min}$ and then quenched with water. Extraction of the mixture with dichloromethane, and subsequent washing of the organic extracts with water, gave a clear solution that was dried (magnesium sulfate). The solvent was removed under reduced pressure to give the product $\mathbf{1 7}$ as a clear oil (11.59 g, 96\%) (Found: C, 57.3; H, 5.0. $\mathrm{C}_{16} \mathrm{H}_{17} \mathrm{BrO}_{3}$ requires C, 57.15; $\left.\mathrm{H}, 5.1 \%\right) ; v_{\max } / \mathrm{cm}^{-1} 3436(\mathrm{O}-\mathrm{H}), 2932(\mathrm{C}-\mathrm{H}), 1601(\mathrm{C}=\mathrm{C}), 1474$ $(\mathrm{C}=\mathrm{C}), 1455(\mathrm{C}=\mathrm{C}) ; \delta_{\mathrm{H}} 1.45$ (3H, d, $J$ 6.4, 1- $\left.\mathrm{CH}_{3}\right), 2.50$ (1H, br. s, OH), 3.80 (3H, s, $\mathrm{OCH}_{3}$ ), $4.98\left(2 \mathrm{H}, \mathrm{s}, \mathrm{CH}_{2}\right), 5.12\left(1 \mathrm{H}, \mathrm{q}, J\right.$ 6.4, 1-H), 7.04 and 7.07 (each $1 \mathrm{H}, \mathrm{d}, J 3.0,4^{\prime}-\mathrm{H}$ and $6^{\prime}-\mathrm{H}$ ), 7.29-7.42 (5H, m, Ph-H); $\delta_{\mathrm{C}} 24.1(\mathrm{C}-2), 61.5\left(\mathrm{OCH}_{3}\right), 65.3(\mathrm{C}-1), 70.6\left(\mathrm{CH}_{2}\right), 112.2\left(\mathrm{C}-6^{\prime}\right)$, 117.2 (C-3'), 118.3 (C-4'), 127.5 (C-2" and C-6"), ${ }^{\text {a }} 128.1$ (C-4"), 128.6 (C-3" and C-5"), ${ }^{\text {a }} 136.5$ (C-1"), 141.1 (C-1'), 147.9 (C-2'), ${ }^{\text {b }} 155.6$ (C-5'); ${ }^{\prime} \mathrm{m} / \mathrm{z} 338$ [M+ $\left.\left({ }^{81} \mathrm{Br}\right), 6 \%\right], 336$ [M $\left.\left.{ }^{+}{ }^{79} \mathrm{Br}\right), 6\right]$, 91 (100), 65 (16).

1-(5'-Benzyloxy-3'-bromo-2'-methoxyphenyl)-1-bromoethane (18). A solution of phosphorus tribromide $(0.286 \mathrm{~g}, 1.06 \mathrm{mmol})$ in dry benzene $(4 \mathrm{~mL})$ was added dropwise to a solution of 
compound 17 (1.02 g, $3.02 \mathrm{mmol})$ in dry benzene $(15 \mathrm{~mL})$, and the mixture stirred for $40 \mathrm{~min}$. The reaction mixture was then quenched with saturated aqueous sodium hydrogen carbonate and extracted with diethyl ether. The organic extracts were washed with saturated aqueous sodium hydrogen carbonate and water and then dried (magnesium sulfate). The solvent was removed under reduced pressure to give the oily product 18 (0.949 g, 78\%) (Found: C, 48.85; H, 3.3. $\mathrm{C}_{16} \mathrm{H}_{16} \mathrm{Br}_{2} \mathrm{O}_{2}$ requires $\mathrm{C}$, 48.25; $\mathrm{H}, 4.05 \%$ ) (Found: $\mathrm{M}^{+}$, 397.9555. $\mathrm{C}_{16} \mathrm{H}_{16}{ }^{79} \mathrm{Br}_{2} \mathrm{O}_{2}$ requires $\mathrm{M}$, 397.9517); $v_{\max } / \mathrm{cm}^{-1} 2932(\mathrm{C}-\mathrm{H}), 1601(\mathrm{C}=\mathrm{C}), 1475(\mathrm{C}=\mathrm{C}), 1455(\mathrm{C}=\mathrm{C}) ; \delta_{\mathrm{H}} 1.94(3 \mathrm{H}, \mathrm{d}, J$ 6.9, 1- $\left.\mathrm{CH}_{3}\right), 3.88\left(3 \mathrm{H}, \mathrm{s}, \mathrm{OCH}_{3}\right), 4.99\left(2 \mathrm{H}, \mathrm{s}, \mathrm{CH}_{2}\right), 5.58(1 \mathrm{H}, \mathrm{q}, J 6.9,1-\mathrm{H}), 7.11\left(2 \mathrm{H}, \mathrm{s}, 4^{\prime}-\mathrm{H}\right.$ and 6'H), 7.30-7.42 (5H, m, Ph-H); $\delta_{\mathrm{C}} 26.5$ (C-2), $41.8(\mathrm{C}-1), 61.3\left(\mathrm{OCH}_{3}\right), 70.7\left(\mathrm{CH}_{2}\right), 113.8\left(\mathrm{C}-6^{\prime}\right)$, 117.4 (C-3'), 119.4 (C-4'), 127.6 (C-2" and C-6"), ${ }^{\mathrm{a}} 128.2$ (C-4"), 128.6 (C-3" and C-5"), ${ }^{\mathrm{a}} 136.2$ (C-1"), 138.8 (C-1'), 147.5 (C-2'), 155.6 (C-5'); ${ }^{b} \mathrm{~m} / \mathrm{z} 320$ [M $\left.{ }^{+}-\mathrm{Br}\left({ }^{81} \mathrm{Br}\right), 8 \%\right], 318\left[\mathrm{M}^{+}-\mathrm{Br}\right.$ $\left.\left({ }^{79} \mathrm{Br}\right), 8\right], 91$ (100), 65 (11).

5'-Benzyloxy-3'-bromo-2'-methoxy- $\alpha^{\prime}$-methylbenzyl 2,2,2-trichloroethanimidate (19). A suspension of sodium hydride ( $6 \mathrm{mg}, 0.25 \mathrm{mmol})$ in dry hexane $(2 \mathrm{~mL})$ was added to a solution of alcohol 17 ( $0.520 \mathrm{~g}, 1.54 \mathrm{mmol})$ and $4 \AA$ molecular sieves in dry tetrahydrofuran $(6 \mathrm{~mL})$. The mixture was stirred for 5 min under nitrogen, and then added, via a canula, to a solution of trichloroacetonitrile $(0.223 \mathrm{~g}, 1.54 \mathrm{mmol})$ and $4 \AA$ molecular sieves in dry diethyl ether $(6 \mathrm{~mL})$ at $0{ }^{\circ} \mathrm{C}$. The reaction mixture was stirred at $0{ }^{\circ} \mathrm{C}$ for $4 \mathrm{~h} 30 \mathrm{~min}$. The solution was concentrated and passed through a silica plug (50\% ethyl acetate-petroleum ether). The crude solution was chromatographed (radial, 10-50\% ethyl acetate-petroleum ether) to give the product 19 ( $0.569 \mathrm{~g}$, 77\%) as white needles, mp 80-81 ${ }^{\circ} \mathrm{C}$ (from hexane) (Found: $\mathrm{C}, 45.1 ; \mathrm{H}, 3.7 ; \mathrm{N}, 2.8$. $\mathrm{C}_{18} \mathrm{H}_{17} \mathrm{BrCl}_{3} \mathrm{NO}_{3}$ requires $\mathrm{C}$, 45.1; $\mathrm{H}, 3.6$; $\mathrm{N}$ 2.9\%) (Found: $\mathrm{M}^{+}$, 478.9455. $\mathrm{C}_{18} \mathrm{H}_{17}{ }^{79} \mathrm{Br}^{35} \mathrm{Cl}_{3} \mathrm{NO}_{3}$ requires $\mathrm{M}, 478.9457) ; v_{\max } / \mathrm{cm}^{-1} 3018(\mathrm{C}-\mathrm{H}), 1665(\mathrm{C}=\mathrm{N}), 1477(\mathrm{C}=\mathrm{C}) ; \delta_{\mathrm{H}} 1.58(3 \mathrm{H}, \mathrm{d}, J$ 6.5, $\left.\alpha^{\prime}-\mathrm{CH}_{3}\right), 3.91\left(3 \mathrm{H}, \mathrm{s}, \mathrm{OCH}_{3}\right), 4.976$ and 4.982 (each $\left.1 \mathrm{H}, \mathrm{d}, J 11.6, \mathrm{CH}_{2}\right), 6.22\left(1 \mathrm{H}, \mathrm{q}, J 6.5, \alpha^{\prime}-\right.$ H), $7.05(1 \mathrm{H}, \mathrm{d}, J$ 3.0, 4'-H), $7.12(1 \mathrm{H}, \mathrm{d}, J$ 3.0, 6'-H), 7.28-7.39 (5H, m, Ph-H), $8.34(1 \mathrm{H}, \mathrm{s}$, $\mathrm{NH}) ; \delta_{\mathrm{C}} 22.0\left(\alpha^{\prime}-\mathrm{CH}_{3}\right), 61.0\left(\mathrm{OCH}_{3}\right), 70.6\left(\mathrm{CH}_{2}\right), 72.6\left(\mathrm{C}-\alpha^{\prime}\right), 91.6\left(\mathrm{CCl}_{3}\right), 111.3\left(\mathrm{C}-6^{\prime}\right), 117.3$ (C-3'), 119.0 (C-4'), 127.5 (C-2" and C-6"'), ${ }^{\mathrm{a}} 128.1$ (C-4"), 128.6 (C-3" and C-5"), ${ }^{\mathrm{a}} 136.4$ (C1'), 137.5 (C-1'), 147.6 (C-2'), ${ }^{\text {b }} 155.7$ (C-5'), ${ }^{\text {b }} 161.2$ (C-1); m/z 320 [M $\left.\mathrm{M}^{+}-\mathrm{Cl}_{3} \mathrm{CONH}_{2}\left({ }^{81} \mathrm{Br}\right), 9 \%\right]$, 318 [M $\left.\mathrm{M}^{+}-\mathrm{Cl}_{3} \mathrm{CONH}_{2}\left({ }^{79} \mathrm{Br}\right), 12\right], 105$ (13), 91 (100), 77 (12), 65 (21).

Methyl ( $\alpha^{\prime} S$ and $\left.R, 2 R\right)$-2-(5'-benzyloxy-3'-bromo-2'-methoxy- $\alpha^{\prime}$ methyl-benzyloxy)propanoate (21). Boron trifluoride diethyl etherate $(4 \mathrm{mg}, 0.03 \mathrm{mmol})$ was added dropwise to a solution of imidate $19(80 \mathrm{mg}, 0.17 \mathrm{mmol})$, methyl $(R)$-lactate $(34 \mathrm{mg}, 0.33 \mathrm{mmol})$ and $4 \AA$ molecular sieves in dry hexane:dry dichloromethane $(5 \mathrm{~mL}, 2: 1)$. The mixture was stirred under nitrogen for $40 \mathrm{~min}$. Solid sodium hydrogen carbonate was added to the reaction and the resulting suspension was filtered through a short plug of silica. The clear solution was then concentrated and chromatographed (radial, 10\% ethyl acetate-petroleum ether) to yield the products 21 as an oily mixture (1:1) of two inseparable diastereoisomers (45 mg, 64\%) (Found: C, 57.1; $\mathrm{H}, 5.2 . \mathrm{C}_{20} \mathrm{H}_{23} \mathrm{BrO}_{5}$ requires C, 56.85; H, 5.5\%); $v_{\max } / \mathrm{cm}^{-1} 2933(\mathrm{C}-\mathrm{H}), 1752(\mathrm{C}=\mathrm{O})$, $1599(\mathrm{C}=\mathrm{C}), 1565(\mathrm{C}=\mathrm{C}), 1469(\mathrm{C}=\mathrm{C}), 1454(\mathrm{C}=\mathrm{C}) ; \delta_{\mathrm{H}}$ (Mixture of two diastereoisomers) 1.28 and 1.42 (each $1 \mathrm{H}, \mathrm{d}, J$ 6.8, 2- $\mathrm{CH}_{3}$ ), 1.437 and 1.445 (each $1 \mathrm{H}, \mathrm{d}, J$ 6.4, $\alpha^{\prime}-\mathrm{CH}_{3}$ ), 3.60, 3.741, 
3.744 and 3.80 (each 3H, s, $\mathrm{CO}_{2} \mathrm{CH}_{3}$ and $\mathrm{ArOCH}_{3}$ ), 3.74-3.84 (1H, m, 2-H), $4.00(1 \mathrm{H}, \mathrm{q}, J$ 6.8, 2-H), 4.86 and 4.93 (each $1 \mathrm{H}, \mathrm{q}, J$ 6.4, $\left.\alpha^{\prime}-\mathrm{H}\right), 5.01-5.03\left(2 \mathrm{H}, \mathrm{m}, \mathrm{CH}_{2}\right), 6.98,7.09,7.11$ and 7.12 (each 1H, d, J 3.0, 4'-H and 6'-H), 7.29-7.43 (10H, m, Ph-H); $\delta_{\mathrm{C}}$ (Mixture of two diastereoisomers) 18.2 (C-3), 19.0 (C-3), $23.2\left(\alpha^{\prime}-\mathrm{CH}_{3}\right), 23.8\left(\alpha^{\prime}-\mathrm{CH}_{3}\right), 51.8\left(\mathrm{CO}_{2} \mathrm{CH}_{3}\right), 51.9$ $\left(\mathrm{CO}_{2} \underline{\mathrm{CH}}_{3}\right), 61.4\left(\mathrm{ArOCH}_{3}\right), 61.5\left(\mathrm{ArOCH}_{3}\right), 70.6$ (2 x CH $\left.\mathrm{CH}_{2}\right), 71.2(\mathrm{C}-2), 71.6(\mathrm{C}-2), 72.3\left(\mathrm{C}-\alpha^{\prime}\right)$, $72.8\left(\mathrm{C}-\alpha^{\prime}\right), 111.9\left(\mathrm{C}-6^{\prime}\right), 112.6\left(\mathrm{C}-6^{\prime}\right), 117.0\left(\mathrm{C}-3^{\prime}\right), 117.4\left(\mathrm{C}-3^{\prime}\right), 119.0\left(\mathrm{C}-4^{\prime}\right), 119.1$ (C-4'), $127.4\left(\mathrm{C}-2^{\prime \prime}\right.$ and $\left.\mathrm{C}-6^{\prime \prime}\right),{ }^{\mathrm{a}} 127.6\left(\mathrm{C}-2^{\prime \prime}\right.$ and C-6" ${ }^{\prime \prime}{ }^{\mathrm{a}} 128.1\left(\mathrm{C}-4^{\prime \prime}\right), 128.2\left(\mathrm{C}-4^{\prime \prime}\right), 128.6\left(\mathrm{C}-3^{\prime \prime}\right.$ and $\mathrm{C}-$

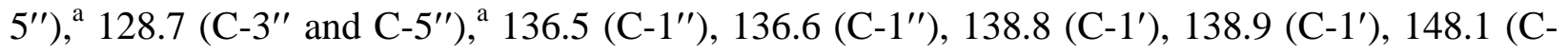
2'), $148.7\left(\mathrm{C}-2^{\prime}\right),{ }^{\mathrm{b}} 155.77\left(\mathrm{C}-5^{\prime}\right),{ }^{\mathrm{b}} 155.83\left(\mathrm{C}-5^{\prime}\right),{ }^{\mathrm{b}} 173.2\left(\mathrm{CO}_{2}\right), 173.9\left(\mathrm{CO}_{2}\right) ; \mathrm{m} / \mathrm{z} 424\left[\mathrm{M}^{+}\left({ }^{81} \mathrm{Br}\right)\right.$, 4\%], 422 [ $\left.\mathrm{M}^{+}\left({ }^{79} \mathrm{Br}\right), 7\right], 337$ (14), 91 (100).

Isobutyl ( $\alpha^{\prime} S$ and $\left.R, 2 R\right)$-2-(5'-benzyloxy-3'-bromo-2'-methoxy- $\alpha^{\prime}$-methyl-benzyloxy)propanoate (22). Boron trifluoride diethyl etherate $\left(\mathrm{BF}_{3} . \mathrm{Et}_{2} \mathrm{O}\right)(23 \mathrm{mg}, 0.16 \mathrm{mmol})$ was added dropwise to a solution of imidate 19 (0.489 g, $1.02 \mathrm{mmol})$ and isobutyl $(R)$-lactate $(0.297 \mathrm{~g}, 2.03 \mathrm{mmol})$ in dry hexane:dry dichloromethane (20 mL, 2:1). The solution was stirred under nitrogen for $1 \mathrm{~h} 15 \mathrm{~min}$. Solid sodium bicarbonate was added to the reaction and the resulting suspension was filtered through a short plug of silica. The clear solution was then concentrated and chromatographed (radial, 10\% ethyl acetatepetroleum ether) to yield an oily mixture of compounds 22 as a 1:1 mixture of diastereoisomers (0.372 g, 79\%) (Found: C, 59.35; H, 6.65. $\mathrm{C}_{23} \mathrm{H}_{29} \mathrm{BrO}_{5}$ requires C, 59.35; H, 6.3\%); $v_{\max } / \mathrm{cm}^{-1}$ $2934(\mathrm{C}-\mathrm{H}), 1748(\mathrm{C}=\mathrm{O}), 1598(\mathrm{C}=\mathrm{C}), 1471(\mathrm{C}=\mathrm{C}) ; \delta_{\mathrm{H}}$ (Mixture of two diastereoisomers) 0.86 and 0.94 (each 6H, d, $J$ 6.7, $\left.\mathrm{CH}\left(\mathrm{CH}_{3}\right)_{2}\right), 1.29$ (3H, d, $J$ 6.9, 2- $\left.\mathrm{CH}_{3}\right), 1.43\left(3 \mathrm{H}, \mathrm{d}, J\right.$ 6.8, 2- $\mathrm{CH}_{3}$ ), 1.44 (3H, d, $J$ 6.4, $\alpha^{\prime}-\mathrm{CH}_{3}$ ), 1.45 (3H, d, $J$ 6.4, $\alpha^{\prime}-\mathrm{CH}_{3}$ ), 1.86 and 1.96 (each $1 \mathrm{H}$, septet, $J$ 6.7, $\left.\mathrm{C} \underline{\mathrm{H}}\left(\mathrm{CH}_{3}\right)_{2}\right), 3.73$ and 3.80 (each $\left.3 \mathrm{H}, \mathrm{s}, \mathrm{OCH}_{3}\right), 3.78(1 \mathrm{H}, \mathrm{q}, J$ 6.9, 2-H), 3.90 (2H, dd, $J 6.6$ and 10.6, $\mathrm{CHC}_{2}$ ), 3.99 (2H, dd, $J 6.6$ and 10.6, $\mathrm{CHCH}_{2}$ ), 4.01 (1H, q, $J$ 6.8, 2-H), 4.89 and 4.93 (each 1H, q, $J$ 6.4, $\alpha^{\prime}-\mathrm{H}$ ), 5.00 (2H, s, $\mathrm{CH}_{2} \mathrm{Ph}$ ), 5.01 and 5.04 (each 1H, d, $J$ 11.8, $\mathrm{CH}_{2} \mathrm{Ph}$ ), 6.99, 7.08, 7.12 and 7.13 (each $1 \mathrm{H}, \mathrm{d}, J 3.1,4^{\prime}-\mathrm{H}$ and $\left.6^{\prime}-\mathrm{H}\right), 7.29-7.43(10 \mathrm{H}, \mathrm{m}, \mathrm{Ph}-\mathrm{H})$; $\delta_{\mathrm{C}}$ (Mixture of two diastereoisomers) 18.3 (C-3), $\left.19.00(\mathrm{C}-3), 19.03\left(\mathrm{CH}\left(\underline{C H}_{3}\right)_{2}\right), 19.1\left(\mathrm{CH}(\underline{\mathrm{CH}})_{3}\right)_{2}\right), 23.2\left(\alpha^{\prime}-\right.$ $\left.\mathrm{CH}_{3}\right), 23.8\left(\alpha^{\prime}-\mathrm{CH}_{3}\right), 27.6\left(\underline{\mathrm{CH}}\left(\mathrm{CH}_{3}\right)_{2}\right), 27.8\left(\underline{\mathrm{CH}}\left(\mathrm{CH}_{3}\right)_{2}\right), 61.4\left(\mathrm{OCH}_{3}\right), 61.5\left(\mathrm{OCH}_{3}\right), 70.5$ $\left(\mathrm{CH}_{2} \mathrm{Ph}\right), 70.6\left(\mathrm{CH}_{2} \mathrm{Ph}\right), 70.8\left(\mathrm{CHCH}_{2}\right), 70.9\left(\mathrm{CHCH}_{2}\right), 71.2(\mathrm{C}-2), 71.6(\mathrm{C}-2), 72.4\left(\mathrm{C}-\alpha^{\prime}\right), 72.8$ (C- $\left.\alpha^{\prime}\right), 111.9\left(\mathrm{C}-6^{\prime}\right), 112.7$ (C-6'), $117.0\left(\mathrm{C}-3^{\prime}\right), 117.3$ (C-3'), 118.8 (C-4'), 119.0 (C-4'), 127.4

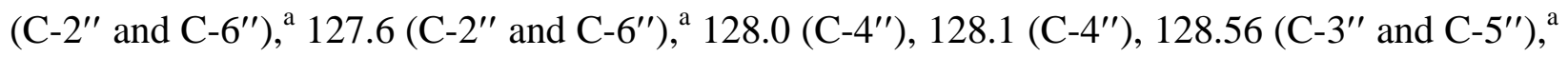
$128.65\left(\mathrm{C}-3^{\prime \prime}\right.$ and C-5"), ${ }^{\mathrm{a}} 136.5\left(\mathrm{C}-1^{\prime \prime}\right), 136.6\left(\mathrm{C}-1^{\prime \prime}\right), 138.9\left(\mathrm{C}-1^{\prime}\right), 139.0\left(\mathrm{C}-1^{\prime}\right), 148.0\left(\mathrm{C}-2^{\prime}\right),{ }^{\mathrm{b}}$ $148.7\left(\mathrm{C}-2^{\prime}\right),{ }^{\mathrm{b}} 155.76\left(\mathrm{C}-5^{\prime}\right),{ }^{\mathrm{b}} 155.83\left(\mathrm{C}-5^{\prime}\right)$, ${ }^{\mathrm{b}} 172.8\left(\mathrm{CO}_{2}\right), 173.5\left(\mathrm{CO}_{2}\right) ; \mathrm{m} / \mathrm{z} 466 \mathrm{M}^{+}\left({ }^{81} \mathrm{Br}\right)$, 5\%], 464 [M $\left.{ }^{+}\left({ }^{79} \mathrm{Br}\right), 9\right], 355$ (12), 253 (11), 207 (16), 91 (100), 73 (34).

Isobutyl ( $\alpha^{\prime} S$ and $\left.R, 2 R\right)$-2-(5'-benzyloxy-3'-chloro-2'-methoxy- $\alpha^{\prime}$-methylben-zyloxy)propanoate (23). A suspension of sodium hydride (21 $\mathrm{mg}, 0.88 \mathrm{mmol}$ ) in dry tetrahydrofuran (2 $\mathrm{mL}$ ) was added to a solution of alcohol 16 (1.29 g, $4.41 \mathrm{mmol})$ and $4 \AA$ molecular sieves (1g) in dry tetrahydrofuran $(20 \mathrm{~mL})$. The reaction was stirred for $5 \mathrm{~min}$ and then added dropwise, via a canula, to a solution of trichloroacetonitrile $(0.96 \mathrm{~g}, 6.65 \mathrm{mmol})$ in dry diethyl ether $(20 \mathrm{~mL})$ at $-5{ }^{\circ} \mathrm{C}$ over $10 \mathrm{~min}$. The reaction was stirred at $-5{ }^{\circ} \mathrm{C}$ for $3 \mathrm{~h} 30 \mathrm{~min}$ and then concentrated and the residue chromatographed (50\% ethyl acetate-petroleum ether) to give crude product that was 
immediately dissolved in dry hexane $(40 \mathrm{~mL})$ and dry dichloromethane $(20 \mathrm{~mL})$. Isobutyl $(R)-$ lactate $(1.29 \mathrm{~g}, 8.82 \mathrm{mmol})$ was added and the mixture stirred at room temperature while a catalytic amount of boron trifluoride diethyl etherate (31 $\mathrm{mg}, 0.22 \mathrm{mmol}$ ) was added. The mixture was stirred for $2 \mathrm{~h} 30 \mathrm{~min}$. Solid sodium hydrogen carbonate (3g) was added and the mixture filtered through silica. The crude solution was chromatographed (radial, 10-30\% ethyl acetate-petroleum ether) to give the products $\mathbf{2 3}$ as an oily mixture (1:1) of two diastereoisomers (0.71 g, 38\%) (Found: C, 65.55; H, 6.70. $\mathrm{C}_{23} \mathrm{H}_{29} \mathrm{ClO}_{5}$ requires $\left.\mathrm{C}, 65.7 ; \mathrm{H}, 6.95 \%\right) ; v_{\max } / \mathrm{cm}^{-1}$ $2970(\mathrm{C}-\mathrm{H}), 1748(\mathrm{C}=\mathrm{O}), 1603(\mathrm{C}=\mathrm{C}), 1574(\mathrm{C}=\mathrm{C}), 1474(\mathrm{C}=\mathrm{C}) ; \delta_{\mathrm{H}}$ (mixture of two diastereoisomers) 0.85-0.88 (12H, m, $\left.\mathrm{CH}\left(\mathrm{CH}_{3}\right)_{2}\right), 1.36$ (6H, d, $J$ 6.6, 2- $\left.\mathrm{CH}_{3}\right), 1.37$ (3H, d, $J$ 6.3, $\left.\alpha^{\prime}-\mathrm{CH}_{3}\right), 1.41\left(3 \mathrm{H}, \mathrm{d}, J\right.$ 6.5, $\left.\alpha^{\prime}-\mathrm{CH}_{3}\right), 1.80-1.91\left(2 \mathrm{H}, \mathrm{m}, \mathrm{C} \underline{\mathrm{H}}\left(\mathrm{CH}_{3}\right)_{2}\right), 3.78$ and 3.80 (each $3 \mathrm{H}, \mathrm{s}$, $\left.\mathrm{OCH}_{3}\right), 3.74-3.89\left(6 \mathrm{H}, \mathrm{m}, \mathrm{CHC} \underline{H}_{2}\right.$ and 2-H), 4.83 and 4.92 (each $\left.1 \mathrm{H}, \mathrm{d}, J 10.6, \mathrm{CH}_{2} \mathrm{Ph}\right), 4.85$ $\left(1 \mathrm{H}, \mathrm{q}, J 6.3, \alpha^{\prime}-\mathrm{H}\right), 4.89\left(1 \mathrm{H}, \mathrm{q}, J\right.$ 6.5, $\left.\alpha^{\prime}-\mathrm{H}\right), 4.96$ and 4.98 (each $1 \mathrm{H}, \mathrm{d}, J 11.0, \mathrm{CH}_{2} \mathrm{Ph}$ ), 6.87 and 6.99 (each $1 \mathrm{H}, \mathrm{d}, J 3.1,4^{\prime}-\mathrm{H}$ and $\left.6^{\prime}-\mathrm{H}\right), 6.91\left(2 \mathrm{H}, \mathrm{d}, J\right.$ 2.0, 4'-H and $\left.6^{\prime}-\mathrm{H}\right), 7.26-7.49(10 \mathrm{H}$, $\mathrm{m}, \mathrm{Ph}-\mathrm{H}) ; \delta_{\mathrm{C}}$ (mixture of two diastereoisomers) $20.8(\mathrm{C}-3),{ }^{\mathrm{a}} 21.4\left(2 \times \mathrm{CH}\left(\underline{\mathrm{C}} \mathrm{H}_{3}\right)_{2}\right), 21.6(\mathrm{C}-3),{ }^{\mathrm{a}}$ $25.6\left(\alpha^{\prime}-\mathrm{CH}_{3}\right), 26.0\left(\alpha^{\prime}-\mathrm{CH}_{3}\right), 30.0\left(\underline{\mathrm{CH}}\left(\mathrm{CH}_{3}\right)_{2}\right), 30.1\left(\underline{\mathrm{CH}}\left(\mathrm{CH}_{3}\right)_{2}\right), 58.1\left(2 \times \mathrm{OCH}_{3}\right), 73.2$ $\left(\mathrm{CH}_{2} \mathrm{Ph}\right),{ }^{\mathrm{b}} 73.3\left(\mathrm{CH}_{2} \mathrm{Ph}\right),{ }^{\mathrm{b}} 73.8(\mathrm{C}-2),{ }^{\mathrm{c}} 73.9(\mathrm{C}-2),{ }^{\mathrm{c}} 74.9\left(\mathrm{C}-\alpha^{\prime}\right),{ }^{\mathrm{d}} 75.2(\mathrm{C}-2),{ }^{\mathrm{d}} 77.9\left(2 \times \mathrm{CHCH}_{2}\right),{ }^{\mathrm{b}}$ 112.8 (C-6'), 113.2 (C-6'), 117.3 (C-4'), 117.6 (C-4'), 130.55 (C-2" and C-6") ${ }^{\prime}$, 130.59 (C-2" and C-6"), 130.68 (C-4"), 130.74 (C-4"), $130.9\left(\mathrm{C}-3^{\prime \prime}\right.$ and C-5"), ${ }^{\mathrm{e}} 131.0$ (C-3" and C-5"), 139.1 (C-1"), 139.4 (C-1"), 141.70 (C-1'), 141.74 (C-1'), 147.7 (C-2'), 148.4 (C-2'), 158.8 (C$\left.5^{\prime}\right),{ }^{\mathrm{f}} 158.9\left(\mathrm{C}-5^{\prime}\right),{ }^{\mathrm{f}} 175.3\left(\mathrm{CO}_{2}\right), 176.0\left(\mathrm{CO}_{2}\right)$ (Signals due to the missing quaternary carbons were lost in the noise owing to the low concentration of the sample used.); $\mathrm{m} / \mathrm{z} 277\left[\mathrm{M}^{+}-\right.$ $\left.\mathrm{OCH}\left(\mathrm{CH}_{3}\right) \mathrm{CO}_{2} \mathrm{CH}_{2} \mathrm{CH}\left(\mathrm{CH}_{3}\right)_{2}\left({ }^{37} \mathrm{Cl}\right), 4 \%\right], 275\left[\mathrm{M}^{+}-\mathrm{OCH}\left(\mathrm{CH}_{3}\right) \mathrm{CO}_{2} \mathrm{CH}_{2} \mathrm{CH}\left(\mathrm{CH}_{3}\right)_{2}\left({ }^{35} \mathrm{Cl}\right), 11\right]$, 201 (11), 184 (24), 91 (100), 65 (14), 57 (13), 46 (23).

\section{( $\left.\alpha^{\prime} R\right)$-5'-Benzyloxy-3'-bromo-2'-methoxy- $\alpha^{\prime}$-methylbenzyl 2,2,2-trichloroethanimidate}

(25). Borane methylsulphide complex $(0.11 \mathrm{~mL}, 10.1 \mathrm{M})$ was added carefully to dry tetrahydrofuran $(8 \mathrm{~mL})$ and the solution added via a canula to a solution of compound $15(0.52 \mathrm{~g}$, $1.55 \mathrm{mmol})$ and CBS-catalyst ${ }^{6,7}(0.15 \mathrm{~mL}, 0.5 \mathrm{M})$ in dry tetrahydrofuran $(8 \mathrm{~mL})$ at $-30{ }^{\circ} \mathrm{C}$. The solution was stirred at $-30{ }^{\circ} \mathrm{C}$ for $1 \mathrm{~h}$, and allowed to warm to room temperature over $1 \mathrm{~h}$. Methanol (2 mL) was added dropwise to the reaction mixture which was stirred for $20 \mathrm{~min}$. The quenched reaction mixture was concentrated, dissolved in diethyl ether, washed with saturated aqueous sodium hydrogen carbonate and saturated aqueous sodium chloride and dried (magnesium sulfate) to give the crude alcohol 24 (94\% enantiomeric excess). The enantiomeric excess was determined by HPLC analysis using 2.5\% 2-propanol in petroleum ether with a Diacel OD column; $t_{R}(S)=29.1 \mathrm{~min},(R)=31.0 \mathrm{~min}$. The crude solution was concentrated, dissolved in dry tetrahydrofuran $(6 \mathrm{~mL})$ and a suspension of sodium hydride (6 $\mathrm{mg}, 0.25 \mathrm{mmol})$ in dry hexane $(2 \mathrm{~mL})$ was added. The mixture was stirred for 5 min and then added via a canula to a solution of freshly distilled trichloroacetonitrile $(0.223 \mathrm{~g}, 1.54 \mathrm{mmol})$ and $4 \AA$ molecular sieves $(0.5 \mathrm{~g})$ in dry diethyl ether $(6 \mathrm{~mL})$ at $0{ }^{\circ} \mathrm{C}$. The reaction was stirred at $0{ }^{\circ} \mathrm{C}$ for $3 \mathrm{~h} 30 \mathrm{~min}$, and then allowed to warm to room temperature over $1 \mathrm{~h}$. The mixture was then concentrated and chromatographed (radial, 10-50\% ethyl acetate-petroleum ether) to yield the product $\mathbf{2 5}(0.21 \mathrm{~g}$, 
28\%). Boron trifluoride diethyl etherate ( $20 \mathrm{mg}, 0.14 \mathrm{mmol}$ ) was added dropwise to a solution of imidate 25 (0.211 g, $0.44 \mathrm{mmol})$, isobutyl $(R)$-lactate $(0.256 \mathrm{~g}, 1.81 \mathrm{mmol})$ and $4 \AA$ molecular sieves $(0.5 \mathrm{~g})$ in dry hexane:dry dichloromethane $(10 \mathrm{~mL}, 2: 1)$. The solution was stirred under nitrogen for $4 \mathrm{~h} 30 \mathrm{~min}$. Solid sodium hydrogen carbonate was added and the resulting suspension was filtered through a short plug of silica. The clear solution was then concentrated and chromatographed (radial, $10 \%$ ethyl acetate-petroleum ether) to yield an oil identified as compounds 22 as a 1:1 mixture of diastereoisomers (71 mg, 35\%).

$\left(\alpha^{\prime} R, 2 R\right)-2-\left(5^{\prime}\right.$-benzyloxy-3'-bromo-2'-methoxy- $\alpha^{\prime}$-methylbenzyloxy)propan-ol 26 and ( $\alpha^{\prime} S$, 2R)-2-(5'-Benzyloxy-3'-bromo-2'-methoxy- $\alpha^{\prime}$-methylbenzyloxy)prop-anol (29). Lithium bromide $(0.156 \mathrm{~g}, 1.80 \mathrm{mmol})$ was added to a solution of sodium borohydride (68 $\mathrm{mg}$, $1.80 \mathrm{mmol})$ in 2-methoxyethyl ether $(6 \mathrm{~mL})$ and the mixture stirred at room temperature for $30 \mathrm{~min}$. A solution of compounds $22(0.559 \mathrm{~g}, 1.20 \mathrm{mmol})$ in 2-methoxyethyl ether (6 $\mathrm{mL})$ was then added to the mixture and the reaction was heated at $95{ }^{\circ} \mathrm{C}$ for $1 \mathrm{~h}$. The reaction was cooled and then quenched with water. Extraction of the quenched reaction mixture with diethyl ether followed by washing of the organic extracts with water gave crude product which was chromatographed (radial, $10-50 \%$ ethyl acetate-petroleum ether-1\% triethylamine) to give two products. The higher $\mathrm{R}_{\mathrm{f}}$ product was identified as compound $29(0.157 \mathrm{~g}, 33 \%)[\alpha]_{\mathrm{D}}-57.5^{\circ}(\mathrm{c}$ 1.0 in $\mathrm{CHCl}_{3}$ ) (Found: C, 57.8; H, 5.95. $\mathrm{C}_{19} \mathrm{H}_{23} \mathrm{BrO}_{4}$ requires C, 57.85; H, 5.9\%); $v_{\max } / \mathrm{cm}^{-1} 3436$ (O-H), $2929(\mathrm{C}-\mathrm{H}), 1599(\mathrm{C}=\mathrm{C}), 1564(\mathrm{C}=\mathrm{C}), 1468(\mathrm{C}=\mathrm{C})$; $\delta_{\mathrm{H}} 1.13$ (3H, d, J 5.9, 2- $\left.\mathrm{CH}_{3}\right), 1.41$ (3H, d, J 6.4, $\left.\alpha^{\prime}-\mathrm{CH}_{3}\right), 2.12\left(1 \mathrm{H}\right.$, br. s, OH), 3.38-3.46 (3H, m, $\mathrm{CH}_{2} \mathrm{OH}$ and 2-H), 3.79 (3H, s, $\left.\mathrm{OCH}_{3}\right), 4.96\left(1 \mathrm{H}, \mathrm{q}, J 6.4, \alpha^{\prime}-\mathrm{H}\right), 5.00\left(2 \mathrm{H}, \mathrm{s}, \mathrm{CH}_{2} \mathrm{Ph}\right), 7.02$ and 7.10 (each $1 \mathrm{H}, \mathrm{d}, J 3.0,4^{\prime}-\mathrm{H}$ and 6'-H), 7.28-7.41 (5H, m, Ph-H); $\delta_{\mathrm{C}} 16.2(\mathrm{C}-3), 23.8\left(\alpha^{\prime}-\mathrm{CH}_{3}\right), 61.6\left(\mathrm{OCH}_{3}\right), 66.8\left(\mathrm{CH}_{2} \mathrm{OH}\right), 69.0$ (C-2), $70.6\left(\mathrm{CH}_{2} \mathrm{Ph}\right), 73.3\left(\mathrm{C}-\alpha^{\prime}\right), 112.4\left(\mathrm{C}-6^{\prime}\right), 117.3\left(\mathrm{C}-3^{\prime}\right), 118.8$ (C-4'), 127.8 (C-2" and C$\left.6^{\prime \prime}\right),{ }^{\mathrm{a}} 128.2\left(\mathrm{C}-4^{\prime \prime}\right), 128.6$ (C-3" and C-5"'), 136.5 (C-1"), 139.4 (C-1'), 148.4 (C-2'), 155.8 (C$\left.5^{\prime}\right) ;{ }^{\mathrm{b}} \mathrm{m} / \mathrm{z} 396$ [M+ $\left({ }^{81} \mathrm{Br}\right), 4 \%$ ], 394 [M $\left.{ }^{+}\left({ }^{79} \mathrm{Br}\right), 5\right], 321$ [( $\left.\left.{ }^{81} \mathrm{Br}\right), 7\right], 319$ [( $\left.\left.{ }^{79} \mathrm{Br}\right), 7\right], 91$ (100), 65 (22), 51 (17). The lower $R_{\mathrm{f}}$ product was identified as compound $26(0.171 \mathrm{~g}, 36 \%)[\alpha]_{\mathrm{D}}+41.6^{\circ}$ (c 1.0 in $\mathrm{CHCl}_{3}$ ) (Found: $\mathrm{M}^{+}$, 394.0767. $\mathrm{C}_{19} \mathrm{H}_{23}{ }^{79} \mathrm{BrO}_{4}$ requires $\mathrm{M}, 394.0780$ ); $v_{\max } / \mathrm{cm}^{-1} 3452$ (O-H), 2932 (C-H), 1599 (C=C), 1566 (C=C), 1468 (C=C); $\delta_{\mathrm{H}} 0.96$ (3H, d, J 6.3, 2- $\left.\mathrm{CH}_{3}\right), 1.41$ (3H, d, $J$ 6.4, $\alpha^{\prime}-\mathrm{CH}_{3}$ ), 1.60 (1H, br. s, $\mathrm{OH}$ ), 3.42-3.65 (3H, m, $\mathrm{CH}_{2} \mathrm{OH}$ and 2-H), 3.80 (3H, s, $\mathrm{OCH}_{3}$ ), $4.94\left(1 \mathrm{H}, \mathrm{q}, J 6.4, \alpha^{\prime}-\mathrm{H}\right.$ ), 5.02 and 5.03 (each $1 \mathrm{H}, \mathrm{d}, J 12.0, \mathrm{CH}_{2} \mathrm{Ph}$ ), 7.04 and 7.10 (each $1 \mathrm{H}, \mathrm{d}, J$ 3.0, 4'-H and 6'-H), 7.29-7.44 (5H, m, Ph-H); $\delta_{\mathrm{C}} 17.3(\mathrm{C}-3), 23.8\left(\alpha^{\prime}-\mathrm{CH}_{3}\right), 61.5$ $\left(\mathrm{OCH}_{3}\right), 65.9\left(\mathrm{CH}_{2} \mathrm{OH}\right), 70.5(\mathrm{C}-2), 70.6\left(\mathrm{CH}_{2} \mathrm{Ph}\right), 74.5\left(\mathrm{C}-\alpha^{\prime}\right), 112.5\left(\mathrm{C}-6^{\prime}\right), 117.1\left(\mathrm{C}-3^{\prime}\right), 118.7$ (C-4'), 127.5 (C-2" and C-6"), ${ }^{a} 128.1$ (C-4"), 128.6 (C-3" and C-5"), 136.5 (C-1"), 140.2 (C1'), 147.9 (C-2'), 155.7 (C-5'); ${ }^{\mathrm{b}} \mathrm{m} / \mathrm{z} 396$ [M $\left.{ }^{+}\left({ }^{81} \mathrm{Br}\right), 5 \%\right], 394$ [M $\left.{ }^{+}\left({ }^{79} \mathrm{Br}\right), 4\right], 321$ [( $\left.\left.{ }^{81} \mathrm{Br}\right), 9\right], 319$ [ $\left.\left({ }^{81} \mathrm{Br}\right), 14\right], 91$ (100), 65 (15).

$\left(\alpha^{\prime} R, \quad 2 R\right)-2-\left(5^{\prime}\right.$-Benzyloxy-3'-bromo-2'-methoxy- $\alpha^{\prime}$-methylbenzyloxy)propan-al (27). A solution of dimethyl sulfoxide $(0.338 \mathrm{~g}, 4.33 \mathrm{mmol})$ in dry dichloromethane $(4 \mathrm{~mL})$ was added dropwise, over $5 \mathrm{~min}$, to a solution of oxalyl chloride $(0.275 \mathrm{~g}, 2.17 \mathrm{mmol})$ in dry dichloromethane $(4 \mathrm{~mL})$ at $-70{ }^{\circ} \mathrm{C}$ under nitrogen, while keeping the mixture below $-65{ }^{\circ} \mathrm{C}$. The mixture was stirred at $-70{ }^{\circ} \mathrm{C}$ for $25 \mathrm{~min}$. A solution of alcohol $26(0.571 \mathrm{~g}, 1.44 \mathrm{mmol})$ in dry 
dichloromethane $(8 \mathrm{~mL})$ was then added over $10 \mathrm{~min}$ while keeping the temperature below $65{ }^{\circ} \mathrm{C}$. Stirring was continued at $-70{ }^{\circ} \mathrm{C}$ for $10 \mathrm{~min}$. Dry N,N-diisopropylethylamine $(0.933 \mathrm{~g}$, $7.22 \mathrm{mmol}$ ) was then added carefully and the mixture stirred for a further $5 \mathrm{~min}$ at $-70{ }^{\circ} \mathrm{C}$ before being allowed to warm to room temperature over $1 \mathrm{~h}$. The reaction was quenched with water and extracted with dichloromethane. The organic extracts were dried (magnesium sulfate) and the solvent removed under reduced pressure. The crude residue was chromatographed (radial, 10$50 \%$ ethyl acetate-petroleum ether-1\% triethylamine) to give the product $27(0.409 \mathrm{~g}, 72 \%)[\alpha]_{\mathrm{D}}$ +91.13 (c 0.94 in $\mathrm{CHCl}_{3}$ ) (Found: C, 57.95; H, 5.45. $\mathrm{C}_{19} \mathrm{H}_{21} \mathrm{BrO}_{4}$ requires $\mathrm{C}, 58.05 ; \mathrm{H}, 5.4 \%$ ) (Found: $\mathrm{M}^{+}$, 392.0638. $\mathrm{C}_{19} \mathrm{H}_{21}{ }^{79} \mathrm{BrO}_{4}$ requires $\mathrm{M}$, 392.0623); $v_{\max } / \mathrm{cm}^{-1} 2931(\mathrm{C}-\mathrm{H}), 1733(\mathrm{C}=\mathrm{O})$, 1599 (C=C), 1565 (C=C), $1472(\mathrm{C}=\mathrm{C})$; $\delta_{\mathrm{H}} 1.15$ (3H, d, $J$ 7.0, 2-CH $\mathrm{CH}_{3}, 1.47$ (3H, d, J 6.4, $\alpha^{\prime}-$ $\left.\mathrm{CH}_{3}\right), 3.66\left(1 \mathrm{H}, \mathrm{dq}, J 1.9\right.$ and 7.0, 2-H), $3.74\left(3 \mathrm{H}, \mathrm{s}, \mathrm{OCH}_{3}\right), 4.89\left(1 \mathrm{H}, \mathrm{q}, J 6.4, \alpha^{\prime}-\mathrm{H}\right), 5.027$ and 5.034 (each $1 \mathrm{H}, \mathrm{d}, J 11.8, \mathrm{CH}_{2}$ ), 7.00 and 7.12 (each $1 \mathrm{H}, \mathrm{d}, J 3.0,4^{\prime}-\mathrm{H}$ and $\left.6^{\prime}-\mathrm{H}\right), 7.29-7.43(5 \mathrm{H}$, m, Ph-H), $9.67\left(1 \mathrm{H}, \mathrm{d}, J\right.$ 1.9, CHO); $\delta_{\mathrm{C}} 15.9(\mathrm{C}-3), 23.7\left(\alpha^{\prime}-\mathrm{CH}_{3}\right), 61.5\left(\mathrm{OCH}_{3}\right), 70.6(\mathrm{C}-2), 72.4$ $\left(\mathrm{CH}_{2}\right), 78.2$ (C- $\left.\alpha^{\prime}\right), 112.4\left(\mathrm{C}-6^{\prime}\right), 117.4\left(\mathrm{C}-3^{\prime}\right), 119.3\left(\mathrm{C}-4^{\prime}\right), 127.4\left(\mathrm{C}-2^{\prime \prime}\right.$ and C-6"), ${ }^{\text {a }} 128.6$ (C4"), 128.7 (C-3"' and C-5"), ${ }^{a} 136.4$ (C-1"), 138.6 (C-1'), 148.5 (C-2'), 155.7 (C-5'), 203.4 (CHO); $\mathrm{m} / \mathrm{z} 394$ [M $\left.{ }^{+}\left({ }^{81} \mathrm{Br}\right), 4 \%\right], 392\left[\mathrm{M}^{+}\left({ }^{79} \mathrm{Br}\right), 3\right], 321\left[\left({ }^{81} \mathrm{Br}\right) 25\right], 319\left[\left({ }^{79} \mathrm{Br}\right) 22\right], 91(100)$, 65 (13).

( $\left.\alpha^{\prime} S, \quad 2 R\right)$-2-(5'-Benzyloxy-3'-bromo-2'-methoxy- $\alpha^{\prime}$-methylbenzyloxy)propan-al (30). A solution of dimethyl sulfoxide $(0.295 \mathrm{~g}, 3.78 \mathrm{mmol})$ in dry dichloromethane $(4 \mathrm{~mL})$ was added dropwise, over $5 \mathrm{~min}$, to a solution of oxalyl chloride $(0.240 \mathrm{~g}, 1.89 \mathrm{mmol})$ in dry dichloromethane $(4 \mathrm{~mL})$ at $-70{ }^{\circ} \mathrm{C}$ under nitrogen, while keeping the mixture below $-65{ }^{\circ} \mathrm{C}$. This was stirred at $-70{ }^{\circ} \mathrm{C}$ for $25 \mathrm{~min}$. A solution of alcohol $29(0.498 \mathrm{~g}, 1.26 \mathrm{mmol})$ in dry dichloromethane $(7 \mathrm{~mL})$ was then added over 10 min while keeping the temperature below $65^{\circ} \mathrm{C}$. The mixture was stirred at $-70{ }^{\circ} \mathrm{C}$ for $10 \mathrm{~min}$. Dry N,N-diisopropylethylamine $(0.814 \mathrm{~g}$, $6.30 \mathrm{mmol}$ ) was then added carefully and the stirring was continued for a further $5 \mathrm{~min}$ at $-70{ }^{\circ} \mathrm{C}$ before being allowed to warm to room temperature over $1 \mathrm{~h}$. The reaction was quenched with water and extracted with dichloromethane. The organic extracts were dried (magnesium sulfate) and the solvent removed under reduced pressure. The crude residue was chromatographed (radial, $10-50 \%$ ethyl acetate-petroleum ether- $1 \%$ triethylamine) to give the product 30 ( $0.425 \mathrm{~g}$, 86\%) $[\alpha]_{\mathrm{D}}-52.0^{\circ}$ (c 1.0 in $\mathrm{CHCl}_{3}$ ) (Found: $\mathrm{C}, 57.8 ; \mathrm{H}, 5.45 . \mathrm{C}_{19} \mathrm{H}_{21} \mathrm{BrO}_{4}$ requires $\mathrm{C}$, 58.05; $\mathrm{H}$, 5.4\%) (Found: $\mathrm{M}^{+}$, 392.0621. $\mathrm{C}_{19} \mathrm{H}_{21}{ }^{79} \mathrm{BrO}_{4}$ requires $\mathrm{M}$, 392.0623); $v_{\max } / \mathrm{cm}^{-1} 2932(\mathrm{C}-\mathrm{H}), 1736$ $(\mathrm{C}=\mathrm{O}), 1599(\mathrm{C}=\mathrm{C}), 1565(\mathrm{C}=\mathrm{C}), 1472(\mathrm{C}=\mathrm{C}) ; \delta_{\mathrm{H}} 1.28\left(3 \mathrm{H}, \mathrm{d}, J\right.$ 6.8, 2- $\left.\mathrm{CH}_{3}\right), 1.46$ (3H, d, $J$ 6.4, $\left.\alpha^{\prime}-\mathrm{CH}_{3}\right), 3.71\left(1 \mathrm{H}, \mathrm{dq}, J 1.4\right.$ and 6.8, 2-H), $3.78\left(3 \mathrm{H}, \mathrm{s}, \mathrm{OCH}_{3}\right), 4.97\left(1 \mathrm{H}, \mathrm{q}, J 6.4, \alpha^{\prime}-\mathrm{H}\right), 5.015$ and 5.023 (each $1 \mathrm{H}, \mathrm{d}, J$ 11.7, $\mathrm{CH}_{2}$ ), 7.04 and 7.12 (each $1 \mathrm{H}, \mathrm{d}, J$ 3.0, $4^{\prime}-\mathrm{H}$ and $6^{\prime}-\mathrm{H}$ ), 7.28-7.42 (5H, m, Ph-H), $9.40\left(1 \mathrm{H}, \mathrm{d}, J\right.$ 1.4, CHO); $\delta_{\mathrm{C}} 15.0(\mathrm{C}-3), 23.6\left(\alpha^{\prime}-\mathrm{CH}_{3}\right), 61.6\left(\mathrm{OCH}_{3}\right), 70.58$ $\left(\mathrm{CH}_{2}\right), 70.63$ (C-2), 78.3 (C- $\left.\alpha^{\prime}\right), 112.4$ (C-6'), $117.4\left(\mathrm{C}-3^{\prime}\right), 119.3$ (C-4'), 127.5 (C-2" and C-6"), ${ }^{\text {a }}$ 128.2 (C-4"), 128.7 (C-3" and C-5"), ${ }^{\text {a }} 136.4$ (C-1"), 138.5 (C-1'), 148.4 (C-2'), 155.8 (C-5'), 202.7 (CHO); $\mathrm{m} / \mathrm{z} 394$ [M $\left.{ }^{+}\left({ }^{81} \mathrm{Br}\right), 2 \%\right], 392$ [M $\left.{ }^{+}\left({ }^{79} \mathrm{Br}\right), 2\right], 321$ (10), 319 (10), 91 (100).

( $\left.\alpha^{\prime} R, 2 R\right)$-2-(3'-Bromo-5'-hydroxy-2'-methoxy- $\alpha^{\prime}$-methylbenzyloxy)propanal (6). A suspension of $10 \%$ palladium on carbon (39 mg) in a solution of aldehyde 27 (78 mg, 
$0.26 \mathrm{mmol})$ in ethyl acetate $(8 \mathrm{~mL})$ was stirred under hydrogen for $30 \mathrm{~min}$. The mixture was filtered through filter aid and then treated with another portion of $10 \%$ palladium on carbon (78 mg). The suspension was stirred under hydrogen for $40 \mathrm{~min}$ and then filtered through filter aid. The solvent was removed under vacuum to give the unstable crude product $6(70 \mathrm{mg})$; $v_{\max } / \mathrm{cm}^{-1} 3392(\mathrm{O}-\mathrm{H}), 2933(\mathrm{C}-\mathrm{H}), 1733(\mathrm{C}=\mathrm{O}), 1603(\mathrm{C}=\mathrm{C}), 1576(\mathrm{C}=\mathrm{C}), 1474(\mathrm{C}=\mathrm{C}), 1457$ $(\mathrm{C}=\mathrm{C}) ; \delta_{\mathrm{H}} 1.23\left(3 \mathrm{H}, \mathrm{d}, J\right.$ 7.1, 2- $\left.\mathrm{CH}_{3}\right), 1.48\left(3 \mathrm{H}, \mathrm{d}, J 6.4, \alpha^{\prime}-\mathrm{CH}_{3}\right), 3.74\left(3 \mathrm{H}, \mathrm{s}, \mathrm{OCH}_{3}\right), 3.75-3.86$ (1H, m, 2-H), 4.90 (1H, q, $J$ 6.4, $\left.\alpha^{\prime}-\mathrm{H}\right), 6.91$ and 7.00 (each 1H, d, $J$ 3.0, 4'-H and 6'-H), 9.68 (1H, d, J 1.7, CHO); $\delta_{\mathrm{C}} 15.7(\mathrm{C}-3), 23.6\left(\alpha^{\prime}-\mathrm{CH}_{3}\right), 61.6\left(\mathrm{OCH}_{3}\right), 71.3(\mathrm{C}-2), 78.2\left(\mathrm{C}-\alpha^{\prime}\right), 112.6$ (C-6'), 117.3 (C-3'), 119.7 (C-4'), 138.3 (C-1'), 148.0 (C-2'), ${ }^{a} 153.2$ (C-5'), ${ }^{a} 203.6$ (CHO); m/z 304 [M $\left.{ }^{+}\left({ }^{81} \mathrm{Br}\right), 5 \%\right], 302$ [M $\left.{ }^{+}\left({ }^{79} \mathrm{Br}\right), 5\right], 286$ (14), 271 (16), 269 (27), 254 (10), 231 (94), 230 (26), 229 (100), 217 (10), 216 (46), 215 (17), 214 (50), 151 (12), 150 (22), 135 (14), 134 (33), 107 (23), 106 (10), 105 (14), 91 (14), 79 (16), 78 (16), 77 (40), 65 (12), 63 (14), 53 (42), 51 (27). ( $\left.\alpha^{\prime} S, 2 R\right)$-2-(3'-Bromo-5'-hydroxy-2'-methoxy- $\alpha^{\prime}$-methylbenzyloxy)propanal (31). A suspension of $10 \%$ palladium on carbon $(72 \mathrm{mg})$ in a solution of aldehyde 30 (0.145 g, $0.37 \mathrm{mmol})$ in ethyl acetate $(8 \mathrm{~mL})$ was stirred under hydrogen for $30 \mathrm{~min}$. The mixture was filtered through filter aid and then treated with another portion of 10\% palladium on carbon (145 $\mathrm{mg}$ ). The suspension was stirred under hydrogen for $30 \mathrm{~min}$ and then filtered through filter aid. The solvent was removed under vacuum to give the unstable crude product $31(0.127 \mathrm{~g})$; $v_{\max } / \mathrm{cm}^{-1} 3402(\mathrm{O}-\mathrm{H}), 2935(\mathrm{C}-\mathrm{H}), 1736(\mathrm{C}=\mathrm{O}), 1604(\mathrm{C}=\mathrm{C}), 1474(\mathrm{C}=\mathrm{C}), 1456(\mathrm{C}=\mathrm{C}) ; \delta_{\mathrm{H}} 1.32$ (3H, d, $J$ 7.0, 2- $\left.\mathrm{CH}_{3}\right), 1.47$ (3H, d, $J$ 6.4, $\left.\alpha^{\prime}-\mathrm{CH}_{3}\right), 3.81(1 \mathrm{H}, \mathrm{dq}, J 1.2$ and 6.8, 2-H), $3.78(3 \mathrm{H}, \mathrm{s}$, $\left.\mathrm{OCH}_{3}\right), 4.97\left(1 \mathrm{H}, \mathrm{q}, J 6.4, \alpha^{\prime}-\mathrm{H}\right), 6.94$ and 6.98 (each $1 \mathrm{H}, \mathrm{d}, J 3.0,4^{\prime}-\mathrm{H}$ and $\left.6^{\prime}-\mathrm{H}\right), 9.51(1 \mathrm{H}, \mathrm{d}, J$ 1.2, $\mathrm{CHO}) ; \delta_{\mathrm{C}} 14.9(\mathrm{C}-3), 23.5\left(\alpha^{\prime}-\mathrm{CH}_{3}\right), 61.7\left(\mathrm{OCH}_{3}\right), 70.6(\mathrm{C}-2), 78.2\left(\mathrm{C}-\alpha^{\prime}\right), 112.8\left(\mathrm{C}-6^{\prime}\right)$, 117.4 (C-3'), 119.8 (C-4'), 138.5 (C-1'), 148.1 (C-2'), ${ }^{a} 153.0$ (C-5'), 202.7 (CHO); m/z 304 [M ${ }^{+}$ $\left.\left({ }^{81} \mathrm{Br}\right), 9 \%\right], 302\left[\mathrm{M}^{+}\left({ }^{79} \mathrm{Br}\right), 10\right], 271\left[\left({ }^{81} \mathrm{Br}\right) 11\right], 269$ [( $\left.\left.{ }^{79} \mathrm{Br}\right) 12\right], 232$ (11), 231 (99), 230 (31), 229 (100), 228 (10), 216 (71), 215 (21), 214 (36), 150 (21), 135 (13), 134 (41), 107 (35), 106 (11), 91 (20), 79 (13), 78 (18), 77 (40), 65 (16), 53 (37), 52 (12), 51 (26), 50 (17).

$(1 R, 3 R, 4 S)$-7-Bromo-4,5-diacetoxy-3,4-dihydro-1,3-dimethyl-8-methoxybezo[c]pyran (28). Titanium(IV) isopropoxide (89 $\mathrm{mg}, 0.31 \mathrm{mmol}$ ) was added to a solution of aldehyde 6 (79 mg, $0.26 \mathrm{mmol})$ in dry dichloromethane $(10 \mathrm{~mL})$ at $0{ }^{\circ} \mathrm{C}$. The reaction was left to stand for $10 \mathrm{~min}$ at $0{ }^{\circ} \mathrm{C}$ and then sonically irradiated at $6-22{ }^{\circ} \mathrm{C}$ for $5 \mathrm{~h} 30 \mathrm{~min}$. The reaction was quenched with saturated aqueous sodium fluoride: saturated aqueous ammonium chloride: dichloromethane (30 $\mathrm{mL}, 1: 1: 1)$ and stirred overnight $(19 \mathrm{~h})$. The aqueous layer was extracted with dichloromethane, the combined organic layers washed with water and then dried (magnesium sulfate). The solvent was removed under reduced pressure to give a pale yellow oil. The crude product 8 was dissolved in dry pyridine $(2 \mathrm{~mL})$ and stirred at room temperature under nitrogen. Acetic anhydride (1.33 g, $13.03 \mathrm{mmol}$ ) was then added to the solution and the reaction stirred overnight $(20 \mathrm{~h})$. The reaction mixture was quenched with water and extracted with diethyl ether. The combined organic layers were washed with hydrochloric acid (1M), water and saturated aqueous sodium chloride. After workup the crude solution was chromatographed (radial, 10-50\% ethyl acetate-petroleum ether) to give the product 28 as a clear oil (52 $\mathrm{mg}, 52 \%$ ) 
(Found: $\mathrm{M}^{+}-\mathrm{CH}_{3} \mathrm{CO}_{2} \mathrm{H}-\mathrm{CH}_{2}=\mathrm{C}=\mathrm{O}$, 286.0038. $\mathrm{C}_{12} \mathrm{H}_{13}{ }^{81} \mathrm{BrO}_{3}$ requires $\mathrm{M}-\mathrm{CH}_{3} \mathrm{CO}_{2} \mathrm{H}-\mathrm{CH}_{2}=\mathrm{C}=\mathrm{O}$, 286.0028); $v_{\max } / \mathrm{cm}^{-1} 1771(\mathrm{C}=\mathrm{O}), 1741(\mathrm{C}=\mathrm{O}), 1467(\mathrm{C}=\mathrm{C}) ; \delta_{\mathrm{H}} 1.23\left(3 \mathrm{H}, \mathrm{d}, J\right.$ 6.6, 3- $\left.\mathrm{CH}_{3}\right), 1.64$ (3H, d, $J$ 6.5, 1- $\left.\mathrm{CH}_{3}\right), 2.09$ (3H, s, 4-OCOCH $), 2.24$ (3H, s, 5- $\left.-\mathrm{OCOCH}_{3}\right), 3.84$ (3H, s, $\mathrm{OCH}_{3}$ ), $4.10(1 \mathrm{H}, \mathrm{dq}, J 5.0$ and 6.6, 3-H), $5.02(1 \mathrm{H}, \mathrm{q}, J 6.5,1-\mathrm{H}), 5.71(1 \mathrm{H}, \mathrm{d}, J 5.0,4-\mathrm{H}), 7.24(1 \mathrm{H}, \mathrm{s}$, 6-H); $\delta_{\mathrm{C}} 17.5\left(3-\mathrm{CH}_{3}\right), 20.2\left(1-\mathrm{CH}_{3}\right), 20.9\left(4-\mathrm{OCOCH}_{3}\right),{ }^{\mathrm{a}} 21.1\left(5-\mathrm{OCOCH}_{3}\right),{ }^{\mathrm{a}} 60.6\left(\mathrm{OCH}_{3}\right), 66.6$ (C-3 and C-4), 68.1 (C-1), 117.0 (C-7), 124.4 (C-4a), ${ }^{\mathrm{b}} 126.1$ (C-6), 136.7 (C-8a), ${ }^{\mathrm{b}} 145.9$ (C-5), ${ }^{\mathrm{c}}$ 150.9 (C-8), ${ }^{\mathrm{c}} 169.0$ (4-CO), ${ }^{\mathrm{d}} 170.5$ (5-CO); ${ }^{\mathrm{d}} \mathrm{m} / \mathrm{z} 329\left(\mathrm{M}^{+}-\mathrm{OCOCH}_{3}, 11 \%\right), 286$ (61), 285 (15), 284 (57), 272 (23), 271 (100), 270 (52), 269 (92).

( $\alpha^{\prime} S$ and $\left.R, 2 R\right)$-2-(5'-Benzyloxy-3'-chloro-2'-methoxy- $\alpha^{\prime}$-methylbenzyloxy)propanol (34). Lithium aluminium hydride (16 mg, $0.42 \mathrm{mmol}$ ) was added to a solution of esters 23 (71 $\mathrm{mg}$, $0.17 \mathrm{mmol}$ ) in dry diethyl ether $(4 \mathrm{~mL})$. The mixture was stirred at room temperature for $1 \mathrm{~h}$ then quenched with water. The quenched reaction mixture was extracted with diethyl ether, dried (magnesium sulfate), and concentrated. The residue was chromatographed (radial, 10\% ethyl acetate-petroleum ether) to give the title compounds $\mathbf{3 4}$ as a mixture (1:1) of two diastereoisomers (42 mg, 71\%) (Found: $\mathrm{M}^{+}$, 352.1258. $\mathrm{C}_{19} \mathrm{H}_{23}{ }^{35} \mathrm{ClO}_{4}$ requires $\mathrm{M}$, 352.1255); $v_{\max } / \mathrm{cm}^{-1} 3453(\mathrm{O}-\mathrm{H}), 2926(\mathrm{C}-\mathrm{H}), 1600(\mathrm{C}=\mathrm{C}), 1459(\mathrm{C}=\mathrm{C}) ; \delta_{\mathrm{H}}$ (mixture of two diastereoisomers) 0.98 (3H, d, $J$ 6.1, 2- $\left.\mathrm{CH}_{3}\right), 1.07$ (3H, d, $J$ 5.7, 2- $\mathrm{CH}_{3}$ ), 1.33 (6H, d, $J$ 6.4, $\alpha^{\prime}-$ $\left.\mathrm{CH}_{3}\right), 1.58(1 \mathrm{H}$, br. s, $\mathrm{OH}), 1.87(1 \mathrm{H}$, br. s, $\mathrm{OH}), 3.35-3.46\left(6 \mathrm{H}, \mathrm{m}, \mathrm{CH}_{2} \mathrm{OH}\right.$ and $\left.2-\mathrm{H}\right), 3.78$ and 3.80 (each $3 \mathrm{H}, \mathrm{s}, \mathrm{OCH}_{3}$ ), 4.86 and 4.90 (each $1 \mathrm{H}, \mathrm{q}, J$ 6.4, $\alpha^{\prime}-\mathrm{H}$ ), 4.96 and 4.97 (each $2 \mathrm{H}, \mathrm{s}$, $\left.\mathrm{CH}_{2} \mathrm{Ph}\right), 6.88-6.93\left(4 \mathrm{H}, \mathrm{m}, 4^{\prime}-\mathrm{H}\right.$ and $\left.6{ }^{\prime}-\mathrm{H}\right), 7.33-7.48(10 \mathrm{H}, \mathrm{m}, \mathrm{Ph}-\mathrm{H}) ; \delta_{\mathrm{C}}$ (mixture of two diastereoisomers) 15.9 (C-3), $17.4(\mathrm{C}-3), 23.6\left(\alpha^{\prime}-\mathrm{CH}_{3}\right), 23.7\left(\alpha^{\prime}-\mathrm{CH}_{3}\right), 55.7\left(\mathrm{OCH}_{3}\right), 65.8(\mathrm{C}-1)$, 66.8 (C-1), 68.8 (C-2), 70.6 (C-2), 73.2 (C- $\left.\alpha^{\prime}\right), 74.5\left(\mathrm{C}-\alpha^{\prime}\right), 75.6\left(\mathrm{CH}_{2} \mathrm{Ph}\right), 75.8\left(\mathrm{CH}_{2} \mathrm{Ph}\right), 110.6$ (C-6'), 110.8 (C-6'), 114.7 (C-4'), 114.9 (C-3'), 128.15, 128.24, 128.3 and 128.6 (C-2" and C-6", C-4", C-3"' and C-5'), 136.8 (C-1'), 139.8 (C-1'), 140.6 (C-1'), 156.3 (C-2' and C-5') (Signals due to the missing quaternary carbons were lost in the noise owing to the low concentration of the sample used.); $\mathrm{m} / \mathrm{z} 352$ [M $\left.\left(\mathrm{M}^{+} \mathrm{Cl}\right), 3 \%\right], 350\left[\mathrm{M}^{+}\left({ }^{35} \mathrm{Cl}\right), 8\right], 276\left[\left({ }^{37} \mathrm{Cl}\right) 10\right], 274\left[\left({ }^{35} \mathrm{Cl}\right) 26\right]$, $186\left[\left({ }^{37} \mathrm{Cl}\right) 36\right], 184\left[\left({ }^{35} \mathrm{Cl}\right) 100\right], 155$ (10), 149 (15).

( $\alpha^{\prime} R$ and $\left.S, 2 R\right)$-2-(5'-Benzyloxy-3'-chloro-2'-methoxy- $\alpha^{\prime}$-methylbenzyloxy)propanal (35). A solution of dimethyl sulfoxide $(0.68 \mathrm{~g}, 8.70 \mathrm{mmol})$ in dry dichloromethane $(4 \mathrm{~mL})$ was added to a solution of oxalyl chloride $(0.55 \mathrm{~g}, 4.33 \mathrm{mmol})$ in dry dichloromethane $(8 \mathrm{~mL})$ at $-70{ }^{\circ} \mathrm{C}$. The mixture was stirred at $-70{ }^{\circ} \mathrm{C}$ for $25 \mathrm{~min}$ and then a solution of alcohols $34(0.30 \mathrm{~g}, 0.86 \mathrm{mmol})$ in dry dichloromethane $(8 \mathrm{~mL}$ ) was added over $10 \mathrm{~min}$ and stirring continued for a further 20 min at $-70{ }^{\circ} \mathrm{C}$. N,N-Diisopropylamine $(1.68 \mathrm{~g}, 13.00 \mathrm{mmol})$ was added over $5 \mathrm{~min}$ and the solution stirred at $-70{ }^{\circ} \mathrm{C}$ for a further $5 \mathrm{~min}$. The mixture was then warmed to room temperature over $1 \mathrm{~h}$, quenched with water and extracted with dichloromethane. The extracts were dried (magnesium sulfate) and concentrated. The residue was chromatographed (radial, 10-50\% ethyl acetate-petroleum ether) to give the products 35 as an inseparable mixture (1:1) of two diastereoisomers (0.26 g, 87\%) (Found: $\mathrm{M}^{+}$, 348.1116. $\mathrm{C}_{19} \mathrm{H}_{21}{ }^{35} \mathrm{ClO}_{4}$ requires $\mathrm{M}, 348.1128$ ); $v_{\max } / \mathrm{Cm}^{-1} 2931(\mathrm{C}-\mathrm{H}), 1737(\mathrm{C}=\mathrm{O}), 1600(\mathrm{C}=\mathrm{C}), 1573(\mathrm{C}=\mathrm{C}), 1474(\mathrm{C}=\mathrm{C}), 1454(\mathrm{C}=\mathrm{C}) ; \delta_{\mathrm{H}}$ (mixture of two diastereoisomers) 1.19 and 1.22 (each 3H, d, $J$ 6.9, 2- $\mathrm{CH}_{3}$ ), 1.36 and 1.39 (each 
$3 \mathrm{H}, \mathrm{d}, J 6.5, \alpha^{\prime}-\mathrm{CH}_{3}$ ), 3.56 and 3.60 (each $1 \mathrm{H}, \mathrm{dq}, J 1.6$ and 6.9, 2-H), 3.78 and 3.80 (each $3 \mathrm{H}, \mathrm{s}$, $\mathrm{OCH}_{3}$ ), 4.80 and 4.86 (each $1 \mathrm{H}, \mathrm{q}, J 6.5, \alpha^{\prime}-\mathrm{H}$ ), 4.88 and 4.95 (each $1 \mathrm{H}, \mathrm{d}, J 10.9, \mathrm{CH}_{2} \mathrm{Ph}$ ), 4.96 (2H, s, $\left.\mathrm{CH}_{2} \mathrm{Ph}\right), 6.88-6.94$ (4H, m, 4'-H and 6'-H), 7.35-7.46 (10H, m, Ph-H), 9.48 (2H, d, J 1.3, $\mathrm{CHO}$ ), 9.59 (2H, d, $J$ 1.6, CHO); $\delta_{\mathrm{C}}$ (mixture of two diastereoisomers) 15.2 (C-3), 15.9 (C-3), $23.3\left(\alpha^{\prime}-\mathrm{CH}_{3}\right), 23.4\left(\alpha^{\prime}-\mathrm{CH}_{3}\right), 55.7\left(\mathrm{OCH}_{3}\right), 70.8(\mathrm{C}-2), 71.9(\mathrm{C}-2), 75.6\left(\mathrm{CH}_{2} \mathrm{Ph}\right), 75.7\left(\mathrm{CH}_{2} \mathrm{Ph}\right)$, 78.0 (C- $\left.\alpha^{\prime}\right), 78.3\left(\alpha^{\prime}-\mathrm{H}\right), 110.5$ (C-6'), 110.8 (C-6'), 115.0 (C-4'), 115.2 (C-4'), 128.3, 128.38, 128.43, and 128.63 (C-2" and C-6", C-4", C-3" and C-5"), 128.61 (C-3'), 136.6 (C-1"), 136.8 (C-1"), 139.0 ( C-1'), 145.7 (C-2' or C-5'), 156.5 (C-2' or C-5'), 202.7 (CHO), 203.3 (CHO); m/z 348 (M+1 12\%), 276 (35), 275 (40), 274 (89), 213 (28), 205 (21), 186 (40), 185 (70), 184 (100), 183 (50), 171 (25), 170 (24), 169 (36), 164 (57), 155 (44), 149 (49), 148 (46).

\section{Acknowledgements}

Receipt of Australian Postgraduate Research Awards (F. J. O. and C. P. T.), an Overseas Postgraduate Research Scholarship (F. J. O.) and financial support from the Senate of Murdoch University are gratefully acknowledged.

\section{References}

1. Giles, R. G. F.; Joll, C. A. J. Chem. Soc., Perkin Trans. 1 1999, 3039.

2. Cameron, D. W.; Cromartie, R. I. T.; Kingston, D. G. I.; Todd, A. R. J. Chem. Soc. 1964, 51.

3. (a) Banville, J; Brassard, P. J. Chem. Soc., Perkin Trans. 1 1976, 1852. (b) Cameron, D. W.; Feutrill, G. I.; Hodder, D. J. J. Chem. Soc., Chem. Commun. 1978, 688. (c) Savard, J.; Brassard, P. Tetrahedron Lett. 1979, 4911. (d) Cameron, D. W.; Conn, C; Feutrill, G. I. Aust. J. Chem. 1981, 34, 1945.

4. Ling, A. R. J. Chem. Soc. 1892, 61, 563.

5. Gopinathan, M. B.; Bhatt, M. V. Indian J. Chem., Sect. B. 1981, 20, 71.

6. (a) Corey, E. J.; Bakshi, R. K. Tetrahedron Lett. 1990, 31, 611. (b) Jones, T. K.; Mohan, J. J.; Xavier, L. C.; Blacklock, T. J.; Mathre, D. J.; Sohar, P.; Jones, E. T. T.; Hoogsteen, K.; Baum, M. W.; Grabowsky, E. J. J. J. Org. Chem. 1991, 56, 763. (c) Mathre, D. J.; Thompson, A. S.; Douglas, A. W.; Hoogsteen, K.; Carroll, J. D.; Corley E. G.; and Grabowski, E. J. J. J. Org. Chem. 1993, 58, 2880.

7. Wallbaum, S.; Martens, J. Tetrahedron: Assymmetry 1992, 3, 1475.

8. (a) Omura,K.; Swern, D. Tetrahedron 1978, 34, 1651. (b) Mancuso, A.J.; Swern, D. Synthesis 1981, 165.

9. Baldwin, J. E.; Haraldsson, G. G. Acta Chem. Scand., Ser.B 1986, 40, 400. 